\title{
INNOVACIONES DE LA INSTRUCCIÓN DE HORMIGÓN ESTRUCTURAL "EHE"
}

\author{
(THE "EHE" CODE INNOVATIONS)
}

Jesús Gómez Hermoso, Dr. Ingeniero de Caminos, Canales y Puertos

FCC Construcción, S. A.

Fecha de recepción: 23-IV-99

ESPAÑA

400-33

\section{RESUMEN}

En el presente artículo se realiza un repaso general de la nueva Instrucción de Hormigón Estructural "EHE". Se describen los aspectos de la misma que suponen una modificación o una nueva aportación, respecto a las anteriores Instrucciones de Hormigón Armado y Hormigón Pretensado.

\section{SUMMARY}

This document make a general review of de new Structural Concrete Instruction "EHE". It describe the aspects that supose a modification or a new contribution in relation to previous Reinforced Concrete and Prestressed Concrete Instructions.

\section{CONSIDERACIONES PREVIAS}

La aprobación de una normativa en cualquier materia supone un cierto "terremoto" en el campo de aplicación de la misma. Si, además, implica unos cambios amplios respecto a las anteriormente existentes, el fenómeno natural mencionado puede alcanzar una elevada intensidad.

Por tanto, se puede afirmar que existe la obligación moral por parte de los autores de la misma de darla a conocer, comentarla, divulgarla y hacerla próxima a todos aquellos que, viéndose afectados por ella, no la conocen tan a fondo, ni en su forma ni en su filosofia. Y esta labor se está realizando. Son muchos los Seminarios, Jornadas y Cursos que se están celebrando por toda la geografía española para desarrollar dicho trabajo. Creo que todos los técnicos debemos agradecer el esfuerzo que se está realizando en este sentido.

Entonces, si existe un texto de la Instrucción, si se están impartiendo una serie de cursos, ¿qué pretende un artículo como éste? El objetivo fundamental es servir de rápida introducción a la lectura del articulado, comentarios y anejos, de tal forma que, una vez conocidos los aspectos que han sido modificados, suprimidos o incorporados como nuevos sea más fácil el acceso a un texto oficial largo y árido, como no podía ser de otro modo. Por tanto, no se plantea como un resumen de la Instrucción, sino como un medio donde se subrayan las principales innovaciones.

Se pretende una exposición aséptica, no crítica, meramente descriptiva. Aunque cualquiera que haya leído el texto puede encontrar algunos aspectos criticables o, simplemente, mejorables, creo que es bueno dejar transcurrir al menos unos meses, contrastar en la práctica diaria del proyecto, la ejecución y el control de las obras y, posteriormente, realizar las aportaciones que cada técnico estime convenientes, subrayando los aspectos positivos o proponiendo modificaciones de los que se han consideradonegativos.

\section{ASPECTOS GENERALES}

Aunque en los apartados siguientes se realice un desarrollo más pormenorizado, en éste se comentan muy breve- 
mente las principales modificaciones llevadas a cabo. Son las siguientes:

- Se unifican en una las anteriores Instrucciones de Hormigón Armado EH-91 (en adelante EH) y Hormigón Pretensado EP-93 (en adelante EP).

- Sustituye de forma directa a las dos Instrucciones citadas, pero presenta una serie de planteamientos (incluso tratamiento de aspectos concretos), que entran en contraposición con otras normativas (EF-96, AE-88, CPI-96, IAP-98, RPX-95, ...)

- Se subraya la importancia de las Certificaciones y los Distintivos de los materiales, medios y empresas.

- Se adopta el Sistema Internacional de unidades.

- Se modifica el planteamiento de las Acciones y sus Combinaciones.

- Se modifican los coeficientes parciales de seguridad.

- Se elevan las características mecánicas mínimas del hormigón, modificándose otras de este material, así como del acero, tanto de armaduras pasivas como activas.

- Se realiza un tratamiento más profundo de la durabilidad, definiendo con más detalle todos aquellos aspectos que pueden incidir en ella.

- Se lleva a cabo una reordenación del cálculo estructural, subrayando tres etapas claras: análisis estructural, cálculo de los Estados Límite Últimos y de Servicio y proyecto de los elementos estructurales.

- En aquellas estructuras o partes de estructuras en que no sea válida la teoría general de flexión, definidas como "regiones D" (de discontinuidad), se plantea como método de cálculo el de las "bielas y tirantes".

- Los Estados Límite Últimos sufren modificación en su planteamiento y desarrollo, suprimiéndose, además, el de Adherencia y presentando el de Esfuerzo rasante.

- Los Estados Límite de Servicio también se modifican, apareciendo como nuevo el de Vibraciones.

- En el título correspondiente a "Ejecución" se recogen algunos aspectos tratados anteriormente en otros, así como nueva información sobre colocación de armadura, fabricación, transporte y curado del hormigón, y descimbrado del mismo.

- El Control de materiales, tanto del hormigón puesto en obra como sobre otros componentes de la estructura, sufre notables modificaciones.
- El Control de ejecución presenta novedades que afectan tanto a la ordenación de la obra como a los puntos a inspeccionar, planteándose también unas tolerancias no tratadas en anteriores Instrucciones.

Los Anejos sufren un notable aumento en número y desarrollo. Las aportaciones más importantes afectan a los siguientes temas:

- Anejo 3. Recomendaciones para la utilización de los cementos.

- Anejo 5. Determinación de la agresividad de aguas y suelos.

- Anejo 7. Recomendaciones para la protección contra el fuego.

- Anejo 8. E. L. de Agotamiento frente a solicitaciones normales.

- Anejo 9. Secciones fisuradas en servicio sometidas a flexión simple.

- Anejo 10. Tolerancias.

- Anejo 11. Hormigones de alta resistencia.

- Anejo 12. Estructuras sometidas a acciones sísmicas.

- Anejo 13. Documento Nacional de Aplicación del Eurocódigo 2.

\section{INTRODUCCIÓN}

\subsection{Certificación y distintivos}

En este primer capítulo debe subrayarse la importancia dada a la certificación y distintivos. Se hace referencia a la futura operatividad de la obligatoriedad del "marcado CE" en los productos de la construcción. Adicional y voluntariamente dichos productos podrán incorporar un "Certificado CC-EHE", otorgado por Organismos españoles y/o Administraciones Públicas, que acredite el cumplimiento de las especificaciones obligatorias de la Instrucción.

Se recogen también los "distintivos" (actuales marcas, sellos, certificados de calidad, ...), reconocidos oficialmente por un Centro Directivo de las Administraciones Públicas.

La importancia de unos y otros se podrá apreciar a medida que se conozca más a fondo la_Instrucción, así como la forma en que se priman (sobre todo, en el control de materiales) los productos que los poseen, frente a los que carecen de ellos. 
Respecto a la obligatoriedad de la certificación de los productos, sólo se contempla como tal una: la adherencia de las barras corrugadas, así como de los alambres corrugados que forman parte de las mallas electrosoldadas y de las armaduras básicas electrosoldadas en celosía.

\subsection{Unidades}

Se adopta de forma sistemática el Sistema Internacional de unidades. En la EP ya se encontraba contemplado de forma muy generalizada, cosa que no se hacía en la $\mathrm{EH}$.

Esta modificación supone, a efectos prácticos, un cambio en las cifras con las que estamos familiarizados que definen las cargas y las tensiones a que estarán sometidos los elementos estructurales y las secciones, así como en el valor de algunas de las características mecánicas de los materiales.

También supone un cambio formal en algunas expresiones y fórmulas que, si bien no ven modificado su planteamiento teórico, el uso de otras unidades modifica el valor de algunos coeficientes.

\subsection{Documentos del Proyecto}

En la descripción de los documentos que ha de comprender todo Proyecto, se hace una mención expresa (no reflejada con la misma intensidad en normas anteriores), sobre el Estudio geotécnico de los terrenos sobre los que la obra se va a ejecutar.

\section{PRINCIPIOS GENERALES Y MÉTODO DE LOS ESTADOSLÍMITE}

\subsection{Situaciones de proyecto}

Se contemplan tres situaciones de proyecto: persistentes, transitorias y accidentales. Su conceptualmente clara separación tiene una importante influencia en la combinación de acciones y en el cálculo y comprobación de los Estados Límite Últimos y de Servicio.

\subsection{El método de los Estados Límite}

Aunque se realicen comentarios concretos en puntos posteriores, aquí conviene subrayar la aparición en la Instrucción del Estado Límite de Agotamiento por Rasante y la desaparición del Estado Límite Último de Adherencia. Debe recordarse que, en cualquier caso, es obligatoria la certificación de adherencia de las barras y alambres corrugados con uso estructural.

Respecto a los Estados Límite de Servicio, se contempla como novedad el Estado Límite de Vibraciones, siendo éstas las que pueden causar inquietud a los usuarios o provocar daños en la estructura o en los equipos que se encuentran sobre la misma.

\subsection{Bases de cálculo orientadas a la durabilidad}

Quizá sea éste uno de los puntos no sólo más novedosos de la Instrucción, sino de los que tiene mayor trascendencia en otros de la misma. Supone la modificación en la definición del tipo de ambiente en que se encuentra una estructura, siendo aquél un parámetro fundamental en la . definición de las características de los materiales y de las disposiciones constructivas.

El tipo de ambiente se define por la combinación de:

-Una de las clases generales de exposición frente a la corrosión de las armaduras, recogidas en la tabla 1 (8.2.2. en EHE).

-Las clases específicas de exposición relativas a otros procesos de degradación, según la tabla 2 (8.2.3.a).

Las clases generales de exposición, a su vez, se pueden ordenar según el tipo de proceso de corrosión: ninguno (I), de origen diferente a los cloruros (II), por corrosión por cloruros de origen marino (III) y por cloruros no marinos (IV).

Las clases específicas de exposición se ordenan, en función del tipo de proceso de corrosión, en ataque químico (débil, medio o fuerte; $\mathrm{Qa}, \mathrm{Qb}$ y $\mathrm{Qc}$, respectivamente), hielo-deshielo $(\mathrm{H})$, por sales fundentes $(\mathrm{F})$ y erosión $(\mathrm{E}$, por abrasión y cavitación). La definición del tipo de ataque químico se encuentra en la tabla 3 (8.2.3.b), obteniéndose en función de los resultados del análisis químico del agua y del suelo en contacto con el hormigón.

\section{ACCIONES}

\subsection{Clasificación de las acciones}

En esta nueva Instrucción las acciones sufren una cierta variación en su clasificación. Así, en función de su naturaleza, se dividen en directas e indirectas y, por su variación en el espacio, en fijas y libres.

Sin embargo, es la clasificación por su variación en el tiempo la que adquiere mayor importancia en la combinación de las mismas, considerándose:

- Permanentes (G): peso propio de la estructura, de elementos embebidos, accesorios y del equipamiento fijo.

- Permanentes de valor no constante $\left(\mathrm{G}^{*}\right)$ : reológicas y pretensado $(\mathrm{P})$.

- Variables (Q): sobrecargas de uso, climáticas y debidas al proceso constructivo.

- Accidentales (A): impactos, explosiones y sísmicas. 
TABLA $1(8.2 .2)$

Clases generales de exposición relativas a la corrosión de las armaduras

\begin{tabular}{|c|c|c|c|c|c|}
\hline \multicolumn{4}{|c|}{ CLASE GENERAL DE EXPOSICIÓN } & \multirow[b]{2}{*}{ DESCRIPCIÓN } & \multirow[b]{2}{*}{ EJEMPLOS } \\
\hline Clase & Subclase & Designación & $\begin{array}{c}\text { Tipo } \\
\text { de proceso }\end{array}$ & & \\
\hline \multicolumn{2}{|c|}{ no agresiva } & 1 & ninguno & $\begin{array}{l}\text { - interiores de edificios, no sometidos } \\
\text { a condensaciones } \\
\text { - elementos de hormigón en masa }\end{array}$ & $\begin{array}{l}\text { - interiores de edificios, protegidos de la } \\
\text { intemperie }\end{array}$ \\
\hline \multirow[t]{2}{*}{ normal } & $\begin{array}{l}\text { humedad } \\
\text { alta }\end{array}$ & Ila & $\begin{array}{l}\text { corrosión de } \\
\text { origen diferente } \\
\text { de los cloruros }\end{array}$ & $\begin{array}{l}\text { - interiores sometidos a humedades } \\
\text { relativas medias altas (>65\%) o a } \\
\text { condensaciones } \\
\text { - exteriores en ausencia de cloruros, } \\
\text { y expuestos a lluvia en zonas con } \\
\text { precipitación media anual superior } \\
\text { a } 600 \mathrm{~mm} \\
\text { - elementos enterrados o sumergidos. }\end{array}$ & $\begin{array}{l}\text { - sótanos no ventilados } \\
\text { - cimentaciones } \\
\text { - tableros y pilas de puentes en zonas con } \\
\text { precipitación media anual superior a } 600 \mathrm{~mm} \\
\text { - elementos de hormigón en cubiertas de edificios }\end{array}$ \\
\hline & $\begin{array}{l}\text { humedad } \\
\text { media }\end{array}$ & IIb & $\begin{array}{l}\text { corrosión de } \\
\text { origen diferente } \\
\text { de los cloruros }\end{array}$ & $\begin{array}{l}\text { - exteriores en ausencia de cloruros, } \\
\text { sometidos a la acción del agua de } \\
\text { lluvia, en zonas con precipitación } \\
\text { media anual inferior a } 600 \mathrm{~mm}\end{array}$ & $\begin{array}{l}\text { - construcciones exteriores protegidas de la } \\
\text { lluvia. } \\
\text { - tableros y pilas de puentes, en zonas de } \\
\text { precipitación media anual inferior a } 600 \mathrm{~mm}\end{array}$ \\
\hline \multirow{3}{*}{ marina } & aérea & Illa & $\begin{array}{l}\text { corrosión por } \\
\text { cloruros }\end{array}$ & $\begin{array}{l}\text { - elementos de estructuras marinas, } \\
\text { - eor encima del nivel de pleamar } \\
\text { - elementos exteriores de estructuras } \\
\text { situadas en las proximidades de la } \\
\text { línea costera (a menos de } 5 \mathrm{~km} \text { ) }\end{array}$ & $\begin{array}{l}\text { - edificaciones en las proximidades de la costa } \\
\text { - puentes en las proximidades de la costa } \\
\text { - zonas aéreas de diques, pantalanes y otras } \\
\text { obras de defensa litoral } \\
\text { - instalaciones portuarias }\end{array}$ \\
\hline & sumergida & IIIb & $\begin{array}{l}\text { corrosión } \\
\text { por cloruros }\end{array}$ & $\begin{array}{l}\text { - elementos de estructuras marinas } \\
\text { sumergidas permanentemente, por } \\
\text { debajo del nivel mínimo de bajamar }\end{array}$ & $\begin{array}{l}\text { - zonas sumergidas de diques, pantalanes y otras } \\
\text { obras de defensa litoral } \\
\text { - cimentaciones y zonas sumergidas de pilas de } \\
\text { puentes en el mar }\end{array}$ \\
\hline & $\begin{array}{c}\text { en zona } \\
\text { de mareas }\end{array}$ & IIIc & $\begin{array}{l}\text { corrosión } \\
\text { por cloruros }\end{array}$ & $\begin{array}{l}\text { - elementos de estructuras marinas } \\
\text { situadas en la zona de carrera de } \\
\text { mareas }\end{array}$ & $\begin{array}{l}\text { - zonas situadas en el recorrido de marea de diques } \\
\text { pantalanes y otras obras de defensa litoral } \\
\text { - zonas de pilas de puentes sobre el mar, situadas } \\
\text { en el recorrido de marea }\end{array}$ \\
\hline \multicolumn{2}{|c|}{$\begin{array}{l}\text { con cloruros de origen } \\
\text { diferente del medio marino }\end{array}$} & IV & $\begin{array}{l}\text { corrosión } \\
\text { por cloruros }\end{array}$ & $\begin{array}{l}\text { - instalaciones no impermeabilizadas } \\
\text { en contacto con agua que presente } \\
\text { un contenido elevado de cloruros, no } \\
\text { relacionados con el ambiente marino } \\
\text { - superficies expuestas a sales de } \\
\text { deshielo no impermeabilizadas }\end{array}$ & $\begin{array}{l}\text { - piscinas } \\
\text { - pilas de pasos superiores o pasarelas } \\
\text { en zonas de nieve } \\
\text { - estaciones de tratamiento de agua }\end{array}$ \\
\hline
\end{tabular}


TABLA2 (8.2.3.a)

Clases específicas de exposición relativas a otros procesos de deterioro distintos de la corrosión

\begin{tabular}{|c|c|c|c|c|c|}
\hline \multicolumn{4}{|c|}{ CLASE ESPECIFICA DE EXPOSICIÓN } & \multirow[b]{2}{*}{ DESCRIPCIÓN } & \multirow[b]{2}{*}{ EJEMPLOS } \\
\hline Clase & Subclase & Designación & $\begin{array}{c}\text { Tipo } \\
\text { de proceso }\end{array}$ & & \\
\hline \multirow{3}{*}{$\begin{array}{l}\text { química } \\
\text { agresiva }\end{array}$} & débil & Qa & ataque químico & $\begin{array}{l}\text { - elementos situados en ambientes con } \\
\text { contenidos de sustancias químicas } \\
\text { capaces de provocar la alteración del } \\
\text { hormigón con velocidad lenta (ver } \\
\text { tabla 8.2.3.b) }\end{array}$ & $\begin{array}{l}\text { - instalaciones industriales, con sustancias } \\
\text { débilmente agresivas según tabla 8.2.3.b } \\
\text { - construcciones en proximidades de áreas } \\
\text { industriales, con agresividad débil según } \\
\text { tabla 8.2.3.b }\end{array}$ \\
\hline & media & $\mathrm{Qb}$ & ataque químico & $\begin{array}{l}\text { - elementos en contacto con agua de mar } \\
\text { - elementos situados en ambientes con } \\
\text { contenidos de sustancias químicas } \\
\text { capaces de provocar la alteración del } \\
\text { hormigón con velocidad media (ver } \\
\text { tabla 8.2.3.b) }\end{array}$ & $\begin{array}{l}\text { - dolos, bloques y otros elementos para diques } \\
\text { - estructuras marinas, en general } \\
\text { - instalaciones industriales con sustancias de } \\
\text { agresividad media según tabla 8.2.3.b } \\
\text { - construcciones en proximidades de áreas indus- } \\
\text { triales, con agresividad media según tabla 8.2.3.b } \\
\text { - instalaciones de conducción y tratamiento de } \\
\text { aguas residuales con sustancias de agresividad } \\
\text { media según tabla 8.2.3.b }\end{array}$ \\
\hline & fuerte & Qc & ataque químico & $\begin{array}{l}\text { - elementos situados en ambientes con } \\
\text { contenidos de sustar cias químicas } \\
\text { capaces de provocar la alteración del } \\
\text { hormigón con velocidad rápida (ver } \\
\text { tabla 8.2.3.b) }\end{array}$ & $\begin{array}{l}\text { - instalaciones industriales,con sustancias de } \\
\text { agresividad alta de acuerdo con tabla 8.2.3.b } \\
\text { - instalaciones de conducción y tratamiento de } \\
\text { aguas residuales, con sustancias de agresividad } \\
\text { alta de acuerdo con tabla 8.2.3.b }\end{array}$ \\
\hline \multirow[t]{2}{*}{ con heladas } & $\begin{array}{l}\text { sin sales } \\
\text { fundentes }\end{array}$ & $H$ & $\begin{array}{l}\text { ataque } \\
\text { hielo-deshielo }\end{array}$ & $\begin{array}{l}\text { - elementos situadós en contacto } \\
\text { frecuente con agua, o zonas con } \\
\text { humedad relativa media ambiental en } \\
\text { invierno superior al } 75 \% \text { y que tengan } \\
\text { una probabilidad anual superior al } 50 \% \\
\text { de alcanzar al menos una vez } \\
\text { temperaturas por debajo de }-5{ }^{\circ} \mathrm{C} \\
\end{array}$ & $\begin{array}{l}\text { - construcciones en zonas de alta montaña } \\
\text { - estaciones invernales }\end{array}$ \\
\hline & $\begin{array}{l}\text { con sales } \\
\text { fundentes }\end{array}$ & $\mathrm{F}$ & $\begin{array}{l}\text { ataque por } \\
\text { sales fundentes }\end{array}$ & $\begin{array}{l}\text { - elementos destinados al tráfico de } \\
\text { vehículos o peatones en zonas con } \\
\text { más de } 5 \text { nevadas anuales o con valor } \\
\text { medio de la temperatura mínima en } \\
\text { los meses de invierno inferior a } 0^{\circ} \mathrm{C} \\
\end{array}$ & $\begin{array}{l}\text { - tableros de puentes o pasarelas en zonas de alta } \\
\text { montaña }\end{array}$ \\
\hline \multicolumn{2}{|c|}{ erosión } & $E$ & $\begin{array}{l}\text { abrasión } \\
\text { cavitación }\end{array}$ & $\begin{array}{l}\text { - elementos sometidos a desgaste } \\
\text { superficial } \\
\text { - elementos de estructuras hidráulicas en } \\
\text { los que la cota piezométrica pueda } \\
\text { descender por debajo de la presión de } \\
\text { vapor del agua }\end{array}$ & $\begin{array}{l}\text { - pilas de puente en cauces muy torrenciales } \\
\text { - elementos de diques, pantalanes y otras obras de } \\
\text { defensa litoral que se encuentren sometidos a } \\
\text { fuertes oleajes } \\
\text { - pavimentos de hormigón } \\
\text { - tuberías de alta presión }\end{array}$ \\
\hline
\end{tabular}


TABLA3 (8.2.3.b)

Clasificación de la agresividad química

\begin{tabular}{|c|c|c|c|c|}
\hline \multirow{3}{*}{$\begin{array}{c}\text { TIPO DE } \\
\text { MEDIO } \\
\text { AGRESIVO }\end{array}$} & \multirow{3}{*}{ PARÁMETROS } & \multicolumn{3}{|c|}{ TIPO DE EXPOSICIÓN } \\
\hline & & $\mathrm{Qa}$ & $\mathrm{Qb}$ & Qc \\
\hline & & $\begin{array}{l}\text { ATAQUE } \\
\text { DÉBIL }\end{array}$ & $\begin{array}{l}\text { ATAQUE } \\
\text { MEDIO }\end{array}$ & $\begin{array}{l}\text { ATAQUE } \\
\text { FUERTE }\end{array}$ \\
\hline \multirow{6}{*}{ AGUA } & VALOR DEL pH & $6,5-5,5$ & $5,5-4,5$ & $<4,5$ \\
\hline & 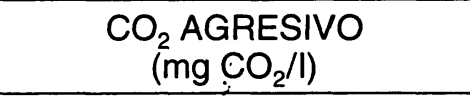 & $15-40$ & $40-100$ & $>100$ \\
\hline & $\begin{array}{l}\text { IÓN AMONIO } \\
\left(\mathrm{mg} \mathrm{NH}_{4}^{+} / \mathrm{l}\right)\end{array}$ & $15-30$ & $30-60$ & $>60$ \\
\hline & $\begin{array}{c}\text { IÓN MAGNESIO } \\
\left(\mathrm{mg} \mathrm{Mg}^{2+} / \mathrm{l}\right)\end{array}$ & $300-1.000$ & $1.000-3.000$ & $>3.000$ \\
\hline & $\begin{array}{l}\text { IÓN SULFATO } \\
\left(\mathrm{mg} \mathrm{SO}_{4}^{2-} / \mathrm{I}\right)\end{array}$ & $200-600$ & $600-3.000$ & $>3.000$ \\
\hline & $\begin{array}{l}\text { RESIDUO SECO } \\
(\mathrm{mg} / \mathrm{l})\end{array}$ & $75-150$ & $50-75$ & $<50$ \\
\hline \multirow{2}{*}{ SUELO } & $\begin{array}{l}\text { GRADO DE ACIDEZ } \\
\text { BAUMANN-GULLY }\end{array}$ & $>20$ & $\left({ }^{\star}\right)$ & $\left({ }^{*}\right)$ \\
\hline & $\begin{array}{c}\text { IÓN SULFATO } \\
\text { (mg SO }{ }_{4}^{2-1} / \mathrm{kg} \text { de suelo seco) }\end{array}$ & $2.000-3.000$ & $3.000-12.000$ & $>12.000$ \\
\hline
\end{tabular}

(*) Estas condiciones no se dan en la práctica

\subsection{Valores de cálculo de las acciones}

Los valores de cálculo de las acciones, tanto para las comprobaciones de los Estados Límite Últimos como de los de Servicio, se obtienen multiplicando los valores representativos por un coeficiente parcial de seguridad, si bien, en el segundo caso, este último es siempre igual a la unidad (salvo para las acciones de pretensado, en cuyo caso se encuentra entre 0,95 y 1,10 ).

Los coeficientes parciales de seguridad de las acciones para las comprobaciones de los Estados Límite Últimos, en función del tipo de acción, de su situación (persistente o transitoria, o accidental) y del tipo de efecto (favorable o desfavorable) se muestran en la tabla 4 (12.1.a).

Entre los valores contemplados debe subrayarse el cambio producido en el caso de situación persistente o transitoria y efecto desfavorable (habitual en un elevado número de cálculos), en los que los coeficientes son 1,35 para acciones permanentes y 1,50 para acciones variables. En las anteriores Instrucciones ambos valores eran iguales a 1,60 .

En la Instrucción EHE no se tiene en cuenta el daño previsible en función del tipo de edificio en caso de colapso de la estructura. Sin embargo, sí se tiene en cuenta, como en Instrucciones anteriores, el tipo de con- trol a efectuar, y con una mayor trascendencia, dada la repercusión que tiene en otros aspectos del proceso constructivo el nuevo planteamiento de dicho control. En la tabla 5 (12.1.b) se muestran los coeficientes, en función del nivel de control de ejecución adoptado, para una situación persistente o transitoria con efecto desfavorable. Es importante observar que el paso de nivel de control intenso a normal supone la elevación de los valores 1,35 y 1,50 , anteriormente citados, a 1,50 y 1,60 , respectivamente.

\subsection{Combinación de acciones}

Se desarrolla una nueva formulación más general, estando formada cada combinación por acciones permanentes, una acción variable determinante y una o varias acciones variables concomitantes.

En estructuras de edificación, tanto para la comprobación de los Estados Límite Últimos, como para los Estados Límite de Servicio, se establecen unas simplificaciones.

\section{MATERIALES Y GEOMETRÍA}

Los coeficientes parciales de seguridad para los materiales son independientes del nivel de control de la obra, adoptándose los indicados en la tabla 6. 
TABLA4(12.1.a)

Coeficientes parciales de seguridad para las acciones, aplicables para la evaluación de los Estados Límite Ultimos

\begin{tabular}{|l|c|c|c|c|}
\hline \multirow{2}{*}{ TIPO DE ACCIÓN } & \multicolumn{2}{|c|}{$\begin{array}{c}\text { Situación persistente } \\
\text { o transitoria }\end{array}$} & \multicolumn{2}{c|}{ Situación accidental } \\
\cline { 2 - 5 } & $\begin{array}{c}\text { Efecto } \\
\text { favorable }\end{array}$ & $\begin{array}{c}\text { Efecto } \\
\text { desfavorable }\end{array}$ & $\begin{array}{c}\text { Efecto } \\
\text { favorable }\end{array}$ & $\begin{array}{c}\text { Efecto } \\
\text { desfavorable }\end{array}$ \\
\hline Permanente & $\gamma_{G}=1,00$ & $\gamma_{G}=1,35$ & $\gamma_{G}=1,00$ & $\gamma_{G}=1,00$ \\
\hline Pretensado & $\gamma_{P}=1,00$ & $\gamma_{P}=1,00$ & $\gamma_{P}=1,00$ & $\gamma_{P}=1,00$ \\
\hline $\begin{array}{l}\text { Permanente de valor no } \\
\text { constante }\end{array}$ & $\gamma_{G^{*}}=1,00$ & $\gamma_{G^{*}}=1,50$ & $\gamma_{G^{*}}=1,00$ & $\gamma_{G^{*}}=1,00$ \\
\hline Variable & $\gamma_{Q}=0,00$ & $\gamma_{Q}=1,50$ & $\gamma_{Q}=0,00$ & $\gamma_{Q}=1,00$ \\
\hline Accidental & - & - & $\gamma_{A}=1,00$ & $\gamma_{A}=1,00$ \\
\hline
\end{tabular}

TABLA 5(12.1.b)

\begin{tabular}{|l|c|c|c|}
\hline \multirow{2}{*}{\multicolumn{2}{|c|}{ TIPO DE ACCIÓN }} & \multicolumn{3}{c|}{ Nivel de control de ejecución } \\
\cline { 2 - 4 } & Intenso & Normal & Reducido \\
\hline Permanente & $\gamma_{G}=1,35$ & $\gamma_{G}=1,50$ & $\gamma_{G}=1,60$ \\
\hline Pretensado & $\gamma_{P}=1,00$ & $\gamma_{P}=1,00$ & - \\
\hline Permanente de valor no constante & $\gamma_{G^{*}}=1,50$ & $\gamma_{G^{*}}=1,60$ & $\gamma_{G^{*}}=1,80$ \\
\hline Variable & $\gamma_{Q}=1,50$ & $\gamma_{Q}=1,60$ & $\gamma_{Q}=1,80$ \\
\hline
\end{tabular}

TABLA 6

\begin{tabular}{|c|c|c|}
\hline Situación de proyecto & Hormigón $\left(\gamma_{\mathrm{c}}\right)$ & Acero pasivo y activo $\left(\gamma_{\mathrm{s}}\right)$ \\
\hline Persistente o transitoria & 1,5 & 1,15 \\
\hline Accidental & 1,3 & 1,0 \\
\hline
\end{tabular}

El coeficiente parcial de seguridad $\gamma_{s}$, permanece con valor 1,15 para cualquier tipo de acero y certificación o sello de calidad, desapareciendo el sello CIETSID y la reducción del citado coeficiente a 1,10 que planteaba la anterior Instrucción.

\section{ANÁLISISESTRUCTURAL}

\subsection{Idealización de la estructura}

Se simplifica el cálculo del ancho eficaz del ala en piezas lineales.
Se especifica que "salvo justificación especial, se considerará como luz de cálculo de las piezas la distancia entre ejes de apoyo".

\subsection{Métodos de cálculo}

Para el análisis global de una estructura se plantean las siguientes metodologías:

- Análisis lineal.

- Análisis no lineal.

- Análisis lineal con redistribución limitada.

- Análisis plástico. 


\subsection{Análisis estructural del pretensado}

Las pérdidas de elementos estructurales pretensados y postensados se tratan de forma independiente.

Se aportan tablas de los valores " $\mu$ " $y$ " $k$ " para pretensado interior con armadura no adherente y para pretensado exterior.

Las pérdidas por acortamiento elástico del hormigón se analizan separadamente para los casos de armaduras pretesa y postesa.

En las pérdidas diferidas del pretensado en armaduras postesas se agrupan en una única expresión matemática las tres causas de la misma, afectando la citada expresión por un denominador que introduce el efecto de la coacción de las deformaciones diferidas debidas a la armadura activa adherente.

\subsection{Estructuras reticulares planas}

Se presentan los cuatro métodos de análisis estructural, ampliando el planteamiento de la EH, en la que se presentaba únicamente el análisis lineal con redistribución limitada. Ésta se mantiene en el $15 \%$.

Se suprime el cálculo simplificado de solicitaciones que figuraba en la tabla 52.2 de la $\mathrm{EH}$.

\subsection{Placas}

En la EHE no se contempla el tratamiento específico de "placas sustentadas en dos bordes paralelos" (artículo $53^{\circ} \mathrm{de} \mathrm{EH}$ ) ni de "placas rectangulares sustentadas en su contorno" (artículo $54^{\circ}$ de EH), recogiéndose en el mismo artículo $22^{\circ}$ el análisis estructural de placas sobre apoyos continuos y aislados.

En los métodos simplificados para el cálculo de esfuerzos de placas sobre apoyos aislados se plantean dos alternativas:

- El Método directo (no explicitado en la EH y sólo válido para cargas verticales).

- El Método de los Pórticos virtuales, cuya hipótesis fundamental es la no interacción entre los citados pórticos virtuales, admite cargas verticales y horizontales y tiene en cuenta la relación entre las rigideces de vigas y soportes.

Respecto a la EH, se han simplificado las tablas de distribución de momentos en la placa y se presenta otra, de distribución de momentos, entre placa y soportes.

\subsection{Regiones D}

Las regiones $\mathrm{D}$ (de discontinuidad), de tratamiento explícito novedoso en esta Instrucción, son estructuras o partes de ellas en que no es válida la teoría general de flexión (no aplicables las hipótesis de Bernouilli-Navier o Kirchhoff).

En la figura 1 (24.1 en EHE) se recogen los tres tipos de discontinuidad:

-Geométrica (cambios geométricos bruscos).

-Estática (cargas concentradas y reacciones).

-Generalizada (estructura en su conjunto, como las ménsulas cortas o las vigas de gran canto).

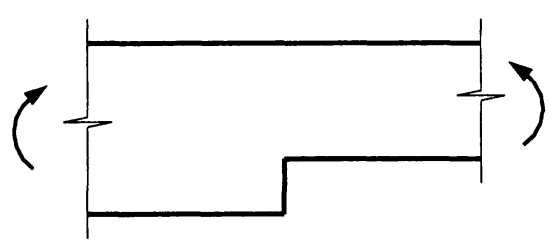

a) DISCONTINUIDAD GEOMÉTRICA

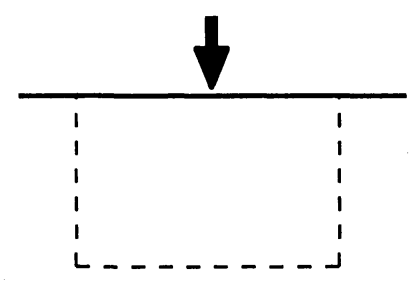

b) DISCONTINUIDAD ESTÁTICA

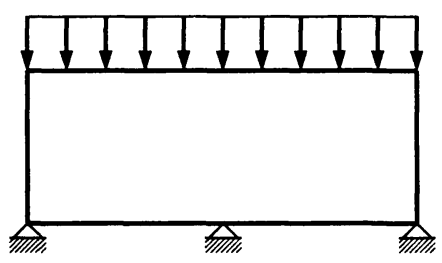

c) DISCONTINUIDAD

GENERALIZADA

Figura 1 
Los tipos de análisis estructural que se plantean son el lineal, el "método de las bielas y tirantes" y el no lineal, recomendándose el segundo.

La aplicación del método de las bielas y tirantes se desarrolla mediante la sustitución de la estructura por barras (bielas para los elementos de hormigón y tirantes para las armaduras). Este método supone un comportamiento plástico perfecto, permitiendo la comprobación de los Estados Límite Últimos.

Sin embargo, el método no permite de forma explícita la comprobación de los Estados Límite de Servicio (sobre todo, fisuración). Para realizar un control indirecto de éstos, se prescribe la consideración del límite elástico de cálculo del acero $\left(f_{y d}\right)$ no superior a $400 \mathrm{~N} / \mathrm{mm}^{2}$.

\subsection{Análisis en el tiempo}

Se realiza un nuevo planteamiento, con tres niveles de análisis:

-Método general

-Método del coeficiente de envejecimiento

-Fórmulas simplificadas.

\section{MATERIALES}

Las características de los materiales se tratan en la Instrucción EHE, básicamente, en tres apartados:

-Título $1^{\circ}$. Bases del proyecto

-Título $3^{\circ}$. Propiedades tecnológicas de los materiales -Título $4^{\circ}$. Cálculo de secciones y elementos estructurales (Datos de los materiales para el proyecto).

Adicionalmente se tratan también, como es lógico, en:

-Título $5^{\circ}$. Ejecución

-Título $6^{\circ}$. Control.

El Título $3^{\circ}$, que se comenta en los próximos subapartados, recoge las propiedades tecnológicas tanto del hormigón como de sus componentes, ya que en los otros dos $\left(1^{\circ}\right.$ y $\left.4^{\circ}\right)$ sólo se tienen en cuenta las de aquél.

\subsection{Cementos}

La Instrucción para la Recepción de Cementos RC-97 es de publicación posterior a las Instrucciones EH y EP. Por tanto, la presente Instrucción EHE ha de adoptar todos aquellos cambios producidos en la RC-97.

El cemento empleado ha de ser de clase resistente 32,5 o superior, utilizándose los tipos de cemento en función del tipo de hormigón que figuran en la tabla 7.

Se define el tipo de endurecimiento del cemento en función de su clase, dividiéndose en lento $(32,5)$, normal $(32,5 R$ y 42,5$)$ y rápido $(42,5 R, 52,5$ y $52,5 R)$.

Se aconseja un almacenamiento máximo de 3, 2 y 1 mes para las clases $32,5,42,5$ y 52,5 , respectivamente.

\subsection{Agua}

El máximo contenido de ión cloro $\left(\mathrm{Cl}^{-}\right)$permitido en agua destinada a la fabricación de hormigón se ha modificado, reduciéndose de 6 a 3 gramos/litro para hormigón armado y elevándose de 0,25 a 1 gramo/litro en hormigón pretensado.

\section{3. Áridos}

Se modifica la serie de tamices para poderse adaptar a la normativa internacional vigente fijándose, además, en $4 \mathrm{~mm}$ ( $5 \mathrm{~mm}$ en $\mathrm{EH}$ y EP) la separación entre áridos fino y grueso.

El tamaño máximo del árido ha de ser inferior a 1,25 veces la distancia entre el borde de la pieza y la vaina o armadura (1,30 en EH y EP), y a 0,4 veces el espesor mínimo de la losa de compresión del forjado (0,5 en EH y EP).

Se modifican también los límites de las sustancias perjudiciales ( $1 \%$ para compuestos totales de azufre y $0,8 \%$ para sulfatos solubles en ácidos).

Se subraya e incrementa la importancia de los análisis petrográficos.

TABLA 7

\begin{tabular}{|l|l|}
\hline \multicolumn{1}{|c|}{ Tipo de hormigón } & \multicolumn{1}{c|}{ Tipo de cemento } \\
\hline Hormigón en masa & $\begin{array}{l}\text { Cementos comunes } \\
\text { Cementos para usos especiales }\end{array}$ \\
\hline Hormigón armado & Cementos comunes \\
\hline Hormigón pretensado & Cementos comunes de los tipos CEM I y CEM II/A-D \\
\hline
\end{tabular}


Respecto a la granulometría y forma del árido se introducen los áridos finos de machaqueo no calizos en ambiente I, IIa y IIb, así como el árido procedente de machaqueo de rocas dolomíticas.

Se define gráficamente y mediante tabulación de sus límites un huso granulométrico para el árido fino.

La forma del árido grueso se expresa y controla mediante el coeficiente de forma (ya contemplado en instrucciones anteriores) y el "índice de lajas", de nueva introducción en ésta, así como una hoja de suministro del árido.

\subsection{Adiciones}

Se contemplan, al igual que en la EH, las cenizas volantes, ampliando y detallando más las especificaciones, y manteniendo la prohibición de su uso en hormigón pretensado.

Se recoge como nueva adición el humo de sílice, detallando las especificaciones que ha de cumplir y limitando su dosificación a un $10 \%$ en peso del cemento para su uso en edificación.

\subsection{Hormigones}

Frente a las anteriores EH y EP en las que se indicaba que "la resistencia a compresión ... ya es por sí misma un índice de la calidad del hormigón", en la EHE se indica que “... no es por sí misma ...", subrayando de esta forma la importancia concedida a los aspectos relacionados con la durabilidad y no sólo con la resistencia a compresión.

Se especifica que la determinación de las características mecánicas, especialmente mediante el ensayo de resistencia a compresión, se realizará mediante rotura de un número de probetas $\geq 2$ ( 3 en $\mathrm{EH}$ y $\mathrm{EP}$ ).

Se indican los tipos de cementos empleados y las relaciones agua/cemento que han de emplearse en un hormigón para que éste pueda calificarse como de endurecimiento rápido, aspecto que se tendrá en cuenta en otros puntos de la Instrucción,como el tiempo mínimo de curado recomendado.

La resistencia característica mínima en función del tipo de hormigón se establece en los siguientes valores:

$\begin{array}{lll}\text { Hormigón en masa } & 20 \mathrm{~N} / \mathrm{mm}^{2} \\ \text { Hormigón armado } & 25 & \text { “ } \\ \text { Hormigón pretensado } & 25 & \end{array}$

Como puede apreciarse, se mantiene el mismo valor que establecía para este último la EP, y se eleva notablemente para el hormigón armado respecto a la $\mathrm{EH}$, fijado en 12,5 como mínimo normativo y siendo el de $17,5 \mathrm{~N} / \mathrm{mm}^{2}(175$ $\mathrm{kp} / \mathrm{cm}^{2}$ ) el mínimo en la práctica, sobre todo, en edificación.
En circunstancias que se consideran especiales (control reducido $u$ obra de ingeniería civil pequeña, edificio de viviendas de no más de 2 plantas y luces menores de 6,00 $\mathrm{m}$ y en vigas menores de $6,00 \mathrm{~m}$ de luz en edificios de hasta cuatro plantas), se establece una resistencia de cálculo $\left(f_{c d}\right)$ no mayor a $10 \mathrm{~N} / \mathrm{mm}^{2}$, utilizándose en cualquier caso un hormigón de resistencia característica $\left(\mathrm{f}_{\mathrm{ck}}\right)$ no inferior a $25 \mathrm{~N} / \mathrm{mm}^{2}$.

Se recomienda, en edificación, el empleo de hormigones con un cono de Abrams no inferior a 6.

\subsection{Armaduras pasivas}

Se suprimen las barras lisas, manteniendo tres tipos de armadura:

-Barras corrugadas.

-Mallas electrosoldadas.

-Armaduras básicas electrosoldadas en celosía (de nueva consideración). En la figura 2 se aprecia su forma, composición y tipo de elementos.

Los diámetros de las barras corrugadas son (en $\mathrm{mm}$ ): 6,8 , $10,12,14,16,20,25,32$ y 40 , suprimiéndose los de 4,5 y $50 \mathrm{~mm}$ y volviendo a contemplar el valor de $14 \mathrm{~mm}$.

Los diámetros nominales de alambres corrugados en mallas electrosoldadas (en mm) son: 5, 5,5, 6, 6,5, 7, 7,5, 8, $8,5,9,9,5,10,10,5,11,11,5,12$ y 14 , desapareciendo los de $4,4,5$ y $13 \mathrm{~mm}$ y contemplando como nuevos los de 10,5 y $11,5 \mathrm{~mm}$. Los alambres de 4 y $4,5 \mathrm{~mm}$ de diámetro no se pueden emplear para comprobación de los Estados Límite Últimos (E.L.U.) a partir del 31-XII-2000.

Los diámetros nominales de alambres, lisos o corrugados, para armaduras básicas electrosoldadas en celosía (en $\mathrm{mm}$ ) son: 5, 6, 7, 8, 9, 10 y 12 , admitiéndose también en elementos transversales de conexión de celosías en forjados unidireccionales los de 4 y $4,5 \mathrm{~mm}$.

En función de las características mecánicas las barras corrugadas se clasifican en dos tipos de acero: B $400 \mathrm{~S}$ y B $500 \mathrm{~S}$, con los mismos valores, respectivamente, que los
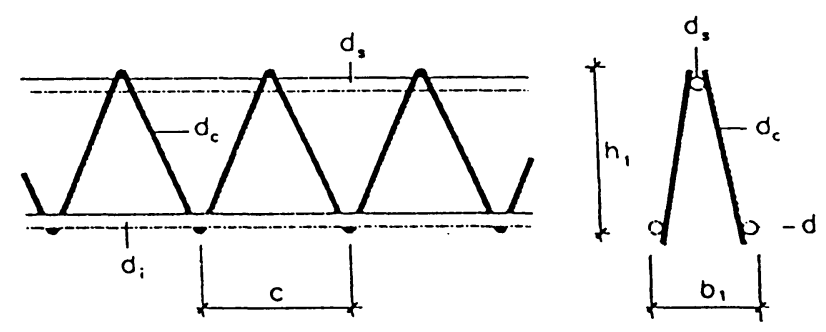

ELEMENTOS LONGITUDINALES: BARRAS O ATLAMBRES CORRUGADOS ELEMENTOS DE CONEXIÓN: BARRAS O ALĀMBBRES (LISOS O CORRUGADOS)

Figura 2 
AEH400S y AEH500S de Instrucciones anteriores. Adicionalmente se considera un nuevo tipo de acero de alta ductilidad, de uso específico para zonas sísmicas, cuya denominación es B 400 SD. La principal diferencia entre éste y los anteriores se encuentra en su valor de deformación $\varepsilon \geq 9 \%$ y en la relación $\mathrm{f}_{\mathrm{s}} / \mathrm{f}_{\mathrm{y}}$ que pasa de 1,05 para los primeros a valores comprendidos entre $1,20 \mathrm{y}$ 1,35 .

Respecto a los ensayos mecánicos desaparece el de doblado simple, permaneciendo el de doblado-desdoblado.

Dejan de contemplarse las mallas lisas, pudiendo estar fabricadas las mallas electrosoldadas con alambres corrugados (igual a EH y EP) o con barras corrugadas.

El tipo de acero para su utilización en estas mallas será el B $500 \mathrm{~T}$, desapareciendo el tipo AEH600T.

Por lo que se refiere al almacenamiento, se indica que una ligera capa de óxido sobre las armaduras pasivas no es perjudicial, pero no se admiten pérdidas por oxidación superiores al $1 \%$ en peso.

\subsection{Armaduras activas}

La denominación de los elementos constituyentes se modifica, pasando el torzal (EP) a considerarse como cordón de 2 ó 3 alambres (EHE) y el cordón ( $>3$ alambres en EP) a cordón de 7 alambres (EHE), no contemplándose el término "cable".

Respecto a las características mecánicas se considera como novedad la resistencia a la tracción desviada.

Los alambres se clasifican, por sus características mecánicas, en cuatro tipos (uno en EP), desápareciendo los de relajación media (R5) y permaneciendo sólo el de relación baja (R2). Sus diámetros nominales son (en mm):
$3,4,5,6,7,7,5,8,9,4$ y 10 , recomendándose el uso de $\phi>4 \mathrm{~mm}$ como alambre aislado.

Los cordones se clasifican, por su carga unitaria máxima, en cuatro valores (uno en EP), para los de 2 ó 3 alambres y en dos (uno en EP), para los de 7 alambres, considerándose, al igual que en los alambres, sólo el tipo de relajación R2.

\subsection{Vainas y accesorios}

Se considera, entre los accesorios, el tubo matriz introducido en el interior de la vaina y se indica, en los comentarios, que las piezas y accesorios de material plástico deberán estar libres de cloruros.

\section{DURABILIDAD}

\subsection{Aspectos generales}

En la Instrucción EHE se subraya la importancia de actuar sobre el proyecto, la ejecución y el uso de las estructuras para mejorar su durabilidad. En especial, en la fase de proyecto, insiste en la selección de la forma estructural de las secciones de hormigón para evitar acumulaciones de agua o el ataque de cualquier agente agresivo.

\subsection{Recubrimiento}

Tanto para las armaduras pasivas como para las activas pretesas, se establecen los siguientes criterios:

-El recubrimiento es la distancia desde el paramento exterior de la sección de hormigón hasta la parte exterior de la armadura principal o del estribo o cerco correspondiente.

-Los recubrimientos mínimos son los que se encuentran recogidos en la tabla $8(37.2 .4)$.

TABLA 8 (37.2.4)

Recubrimientos mínimos

\begin{tabular}{|c|c|c|c|c|c|c|c|c|c|c|c|}
\hline \multirow{2}{*}{$\begin{array}{c}\text { Resistencia } \\
\text { característica } \\
\text { del hormigón } \\
{\left[\mathrm{N} / \mathrm{mm}^{2}\right]}\end{array}$} & \multirow{2}{*}{$\begin{array}{l}\text { Tipo de } \\
\text { elemento }\end{array}$} & \multicolumn{10}{|c|}{$\begin{array}{l}\text { RECUBRIMIENTO MÍNIMO [mm] } \\
\text { SEGÚN LA CLASE DE EXPOSICIÓN (**) }\end{array}$} \\
\hline & & 1 & $\mathrm{Ila}$ & Ilb & IIIa & IIIb & IIIc & IV & Qa & $\mathrm{Qb}$ & Qc \\
\hline \multirow[b]{2}{*}{$25 \leqslant f_{c k}<40$} & general & 20 & 25 & 30 & 35 & 35 & 40 & 35 & 40 & $\left({ }^{*}\right)$ & $\left({ }^{*}\right)$ \\
\hline & $\begin{array}{l}\text { elementos } \\
\text { prefabricados } \\
\text { y láminas }\end{array}$ & 15 & 20 & 25 & '30 & 30 & 35 & 30 & 35 & $(*)$ & $\left({ }^{\star}\right)$ \\
\hline \multirow[b]{2}{*}{$f_{c k} \geqslant 40$} & general & 15 & 20 & 25 & 30 & 30 & 35 & 30 & 35 & $(*)$ & $\left({ }^{*}\right)$ \\
\hline & $\begin{array}{l}\text { elementos } \\
\text { prefabricados } \\
\text { y láminas }\end{array}$ & 15 & 20 & 25 & 25 & 25 & 30 & 25 & 30 & $(*)$ & $(*)$ \\
\hline
\end{tabular}

(*) El proyectista fijará el recubrimiento al objeto de que se garantice adecuadamente la protección de las armaduras frente a la acción agresiva ambiental.

(**) En el caso de clases de exposición H, F o E, el espesor del recubrimiento no se verá afectado. 
-El recubrimiento nominal, que es el que ha de recoger el proyecto y ejecución de la obra, se obtiene sumándole al mínimo el margen de recubrimiento $\left(r_{n o m}=r_{\min }+D r\right)$, siendo este último (Dr):

- 0 (elemento prefabricado, con control intenso de ejecución)

- $5 \mathrm{~mm}$ (elemento in situ, con control intenso de ejecución)

- $10 \mathrm{~mm}$ (resto)

-En las secciones con recubrimiento superior a $50 \mathrm{~mm}$ (40 mm en EH y EP) se indica la posible conveniencia de disponer una malla de reparto.

Se establecen ciertos criterios sobre los separadores, tanto sobre su disposición en la obra (Capítulo XIII. Ejecución), como sobre las características de los materiales que los constituyen y de los que se encuentran prohibidos.

\subsection{Contenido de agua y cemento}

El contenido mínimo de cemento en función del tipo de hormigón se establece en los siguientes valores $\left(\mathrm{kg} / \mathrm{m}^{3}\right)$ :

$\begin{array}{lcc} & \text { EP/EH } & \text { EHE } \\ \text { Hormigón en masa } & 150 & 200 \\ \text { H. ligeramente armado } & 200 & - \\ \text { Hormigón armado } & 250 & 250 \\ \text { Hormigón pretensado } & 250 & 275\end{array}$

Como puede apreciarse se incrementan las cuantías mínimas para hormigón en masa y hormigón pretensado y desaparece el tratamiento de hormigón ligeramente armado, que pasa a considerarse como armado y, por tanto, también con un incremento en la cuantía de cemento.

En la tabla 9 (37.3.2.a) se encuentran reflejadas las máximas relaciones agua/cemento y las mínimas cuantías de cemento en función del tipo de hormigón y de la clase de exposición del elemento estructural. Esto da lugar a que en una misma estructura existan elementos con hormigones con distintas dosificaciones (aunque se les exija la misma resistencia característica).

La tabla 10 (37.3.2.b) recoge las resistencias mínimas compatibles con los requisitos de durabilidad. La referencia a esta tabla se encuentra en los comentarios del artículo $37^{\circ}$ de la EHE, por tanto, se debe entender que no es de obligatorio cumplimiento. Sin embargo, con las exigencias de la tabla 9 es fácil obtener los valores de la tabla 10 .

\subsection{Resistencia frente a agresiones físico-químicas}

Se presentan una serie de apartados en los que se recogen de forma más específica el tratamiento frente a la helada, a los sulfatos, al agua de mar, a la erosión y a la reacción árido-álcali.

En concreto, respecto a la resistencia a los sulfatos se indica que el cemento deberá poseer la característica adicional de resistencia a los sulfatos (SR) cuando el contenido de ion sulfato $\left(\mathrm{SO}_{4}{ }^{\circ}\right.$ ) en agua sea $\geq 600 \mathrm{mg} / \mathrm{l}$ (400 en $\mathrm{EH}$ ) y en suelos $\geq 3.000 \mathrm{mg} / \mathrm{l}$ (igual en $\mathrm{EH}$ ).

\section{DATOS DE LOS MATERIALES PARA EL PRO- YECTO}

Conocidas ya las características tecnológicas de los materiales, en este punto se detallan las correspondientes a los datos para el proyecto.

\subsection{Características de los aceros}

El diagrama tensión-deformación del acero para armaduras pasivas ha sufrido una ligera variación, disponiendo una pendiente no nula para el tramo comprendido entre $f_{y k} y$ $f_{\text {máx }}$.

Sobre la relajación del acero para armaduras activas, en el apartado 38.9, en los comentarios, se aporta una tabla que permite estimar las pérdidas por este concepto.

Se establece una máxima variación de la tensión para definir las características de fatiga de las armaduras activas y pasivas, haciéndose una referencia a las citadas características para los dispositivos de anclaje y empalme de la armadura activa.

\subsection{Características del hormigón}

Los hormigones se tipifican según el siguiente formato:

$$
\mathrm{T}-\mathrm{R} / \mathrm{C} / \mathrm{TM} / \mathrm{A}
$$

siendo:

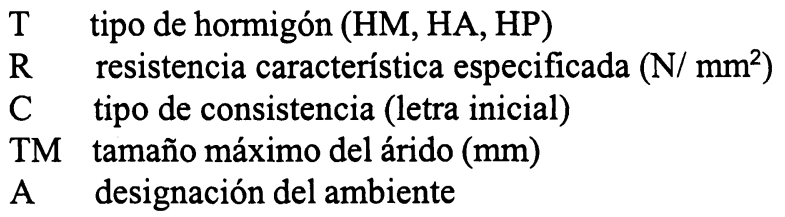

La serie recomendada de resistencia característica especificada es la siguiente:

$20,25,30,35,40,45$ y 50 (resistencia a compresión a 28 dias, expresada en $\mathrm{N} / \mathrm{mm}^{2}$ ). 
TABLA 9 (37.3.2.a)

Máxima relación agua/cemento y mínimo contenido de cemento

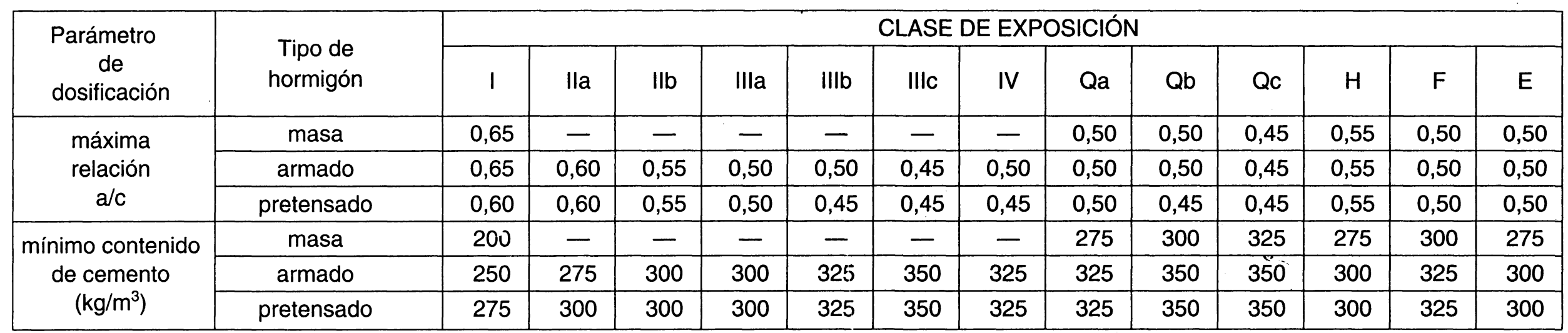

TABLA $10(37.3 .2 . b)$

Resistencias mínimas compatibles con los requisitos de durabilidad

\begin{tabular}{|c|c|c|c|c|c|c|c|c|c|c|c|c|c|c|}
\hline \multirow{2}{*}{$\begin{array}{c}\text { Parámetro } \\
\text { de } \\
\text { dosificación }\end{array}$} & \multirow{2}{*}{$\begin{array}{c}\text { Tipo de } \\
\text { hormigón }\end{array}$} & \multicolumn{13}{|c|}{ CLASE DE EXPOSICIÓN } \\
\hline & & 1 & Ila & $\mathrm{Ilb}$ & IIIa & Illb & IIIC & IV & Qa & $\mathrm{Qb}$ & Qc & $H$ & $\mathrm{~F}$ & $E$ \\
\hline \multirow{2}{*}{$\begin{array}{c}\text { resistencia } \\
\text { mínima } \\
\left(\mathrm{N} / \mathrm{mm}^{2}\right)\end{array}$} & masa & 20 & - & 一 & - & 一 & - & 一 & 30 & 30 & 35 & 30 & 30 & 30 \\
\hline & pretensado & 25 & 25 & 30 & 30 & 35 & 35 & 35 & 30 & 35 & 35 & 30 & 30 & 30 \\
\hline
\end{tabular}


En la obtención del valor de la resistencia de cálculo del hormigón se ha suprimido la reducción del $10 \%$ por hormigonado vertical.

El módulo de deformación longitudinal del hormigón se obtiene en función de la resistencia media a compresión del mismo, aportándose sendas tablas de coeficientes correctores que dependen de la naturaleza del árido y de la edad y tipo de endurecimiento.

Varía la formulación de la retracción del hormigón y se presentan una serie de valores tabulados en función de la humedad relativa, el espesor medio y el tiempo.

El cálculo de la fluencia del hormigón también modifica su formulación y presenta unos valores tabulados en función de los mismos parámetros.

\section{CAPACIDADRESISTENTE DE BIELAS, TIRAN- TES YNUDOS}

En el artículo $40^{\circ}$ se trata este tema, fijándose los criterios de aplicación en el proyecto y cálculo de estructuras. Aunque, al igual que el resto de los temas relativos a cálculo, no es este documento el lugar más adecuado para su explicación detallada, sí se recogen algunas notas sobre el mismo.

El "método de bielas y tirantes" es adecuado para secciones y elementos de hormigón estructural que se encuentren tanto en regiones $\mathrm{B}$ como regiones $\mathrm{D}$ (aunque en esta Instrucción se preconicen, sobre todo, para estas últimas).

Los elementos del modelo son:

-Tirantes (armaduras activas o pasivas)

-Bielas (campo de compresiones)

-Nudos (zona de intersección de bielas y tirantes)

La capacidad resistente de los tirantes se encuentra definida por la de las armaduras que modelizan; la de las bielas se define según los casos estudiados (en zonas con estados de compresión uniaxial, con fisuración oblicua o paralela a la misma biela, con armaduras comprimidas, hormigón confinado o con interferencias de vainas con armaduras activas); y la de los nudos en función de su estado multicomprimido o con tirantes anclados.

\section{ESTADOS LÍMITE ÚLTIMOS}

Los cálculos relativos a los ELU introducen una serie de modificaciones importantes en su desarrollo. En este documento se van a comentar aquéllos de tipo más general, sin entrar en detalle en el proceso completo ni en la formulación. Igualmente, se llamará la atención sobre aquellos aspectos de empleo más general por usuarios del hormigón estructural no expertos en cálculo (cuantías mínimas, separaciones máximas de armaduras, ...).

\subsection{Estado Límite de Agotamiento frente a solicita- ciones normales}

Se realiza una modificación en el planteamiento general del método del momento tope, aportándose en el Anejo $\mathrm{n}^{\circ} 8$ las fórmulas simplificadas para hormigón armado.

Para considerar el efecto de confinamiento en el hormigón se recomienda la aplicación del método de bielas y tirantes.

Se modifican algunas separaciones máximas de armadura como, por ejemplo, la de la pasiva longitudinal resistente (de $50 \mathrm{~cm}$ en $\mathrm{EP}$ a $30 \mathrm{~cm}$ en $\mathrm{EHE}$ ).

Se subraya que en zonas de solapo o doblado puede ser necesario aumentar la armadura transversal.

Las cuantías geométricas mínimas permanecen iguales para pilares y vigas, incrementándose en losas y en muros. En éstos se indica que la armadura horizontal mínima puede reducirse a la mitad si se disponen juntas verticales de contracción con una separación máxima de 7,50 m.

\subsection{Estado Límite de Inestabilidad}

Para la comprobación de soportes aislados se incorpora, en comentarios, un breve desarrollo matemático del Método general de cálculo.

Se incorpora también -como novedad- un planteamiento del método aproximado para los esfuerzos de flexión compuesta esviada.

\subsection{Estado Límite de Agotamiento frente a cortante}

Se establece como método general de cálculo el de bielas y tirantes. Siguiendo el Eurocódigo 2, se plantea el método de inclinación variable de las bielas, sustituyendo al método normalizado $\left(\theta=45^{\circ}\right)$ de la $\mathrm{EH}$.

En las disposiciones relativas a las armaduras, se modifica la separación de estribos, llevándolos hasta los apoyos. También se modifica el "decalaje" de las armaduras longitudinales pasando a ser una función de los parámetros $\mathrm{z}, \theta, \alpha, \mathrm{V}_{\text {su }} \mathrm{y} \mathrm{V}_{\text {rd }}$.

\subsection{Estado Límite de Agotamiento por torsión en elementos lineales}

Se realiza una nueva definición de espesor eficaz $\left(\mathrm{h}_{\mathrm{e}}\right)$ para la comprobación de este ELU, así como de la separación de los cercos (que resulta menor que en la EHE).

Sobre la interacción entre torsión y otros esfuerzos, mientras en la EH se planteaba combinada con el esfuerzo de flexión, en la EHE se hace combinada con flexión y axil, y combinada con el esfuerzo cortante. 


\subsection{Estado Límite de Punzonamiento}

Para la comprobación de este ELU se amplía el área crítica, pasando de $0,5 \mathrm{~d}$ a $2 \mathrm{~d}$ la distancia entre el perímetro de ésta y el del soporte, no llegando dicho perímetro, en los pilares medianeros y de esquina, hasta el borde exterior del forjado. Se plantea también la comprobación de armadura en la zona exterior a la armadura de punzonamiento.

\subsection{Estado Límite de Agotamiento por esfuerzo rasante en juntas entre hormigones}

Este ELU se presenta como novedad en la EHE, planteándose la tensión rasante en función de la cohesión entre hormigones, la armadura existente y el rozamiento entre aquéllos.

Se establece un espesor mínimo de hormigón de $50 \mathrm{~mm}$ a cada lado de la junta, y se realiza el cálculo de la armadura distinguiendo entre junta frágil y dúctil.

\subsection{Estado Límite de Fatiga}

Se presenta nuevo como tratamiento explícito de la fatiga, realizándose una comprobación separada de hormigón y acero. Para el primero da criterios generales, no criterios de comprobación, siendo las acciones consideradas las cargas permanentes y la sobrecarga de fatiga.

Para el acero aporta criterios en función de las características de fatiga de las armaduras, señalando como otros más rigurosos los derivados de la mecánica de fractura. Las acciones consideradas son únicamente las de la sobrecarga de fatiga.

\section{ESTADOS LÍMITE DE SERVICIO}

\subsection{Estado Límite de Fisuración}

Las clases I, II y III consideradas en la Instrucción EP se suprimen en esta EHE.

Para la fisuración por solicitaciones normales se establecen unos valores máximos de abertura de fisura en función de la clase de exposición y del tipo de hormigón (armado o pretensado), empleando diferentes tipos de acciones de comprobación para ambos hormigones.

La limitación de la fisuración debida a esfuerzo cortante se controla con la separación máxima de estribos, determinada en función de dicho esfuerzo, $\mathrm{V}_{\mathrm{rd}}, \mathrm{V}_{\mathrm{cu}}, \mathrm{d}, \alpha$.

Para la limitación de la fisuración por torsión se siguen los mismos criterios de separación de armaduras transversales que en EP.

\subsection{Estado Límite de Deformación}

En los comentarios se establecen, para edificaciones normales, como valores máximos de las flechas total y activa, respectivamente, $\mathrm{L} / 250$ y L/400 (ó $1 \mathrm{~cm}$ ).

Para elementos solicitados a flexión simple o compuesta, se plantean como métodos de cálculo el general (análisis estructural paso a paso en el tiempo mediante doble integración de curvaturas) y el simplificado. Este último, aplicable a vigas y losas de hormigón armado, considerando la flecha compuesta por la suma de una instantánea y una diferida, debida a las cargas permanentes.

Para elementos de hormigón armado con acero $f_{y k}=500$ $\mathrm{N} / \mathrm{mm}^{2}$ no será preciso efectuar la comprobación de la deformación si la relación luz/canto útil $(1 / d)$ es superior a la que se recoge en la tabla 11 (50.2.2.1), siguiendo una filosofía similar a la planteada en la Instrucción EF-96 para forjados.

Para el cálculo de la flecha instantánea se preconiza la inercia constante en toda la longitud de la viga.

Se realiza también un tratamiento de los elementos solicitados a torsión y a tracción pura.

\subsection{Estado Límite de Vibraciones}

Planteado como novedad en la EHE respecto a las anteriores $\mathrm{EH}$ y $\mathrm{EP}$, se indica que, en general, para cumplir el Estado Límite de Vibraciones debe proyectarse la estructura para que las frecuencias naturales de vibración se aparten suficientemente de ciertos valores críticos. Se aportan unos valores mínimos de la citada frecuencia en función del tipo de uso de la construcción.

\section{ELEMENTOSESTRUCTURALES}

En este apartado se comentarán aquellos artículos de la $\mathrm{EHE}$, referidos a elementos estructurales, que hayan sufrido alguna modificación significativa, no haciéndolo de aquellos otros cuyos cambios no lo sean (hormigón en masa, vigas, muros, láminas, ...).

\subsection{Forjados}

La redacción del artículo respecto a los forjados y su referencia a la Instrucción de forjados vigente es semejante a la EH. Sin embargo, en esta EHE se tratan algunos aspectos de los mismos de forma distinta a como se hace en la EF-96. Estas diferencias deben ser tenidas en cuenta en el proyecto y ejecución de obras con este tipo de elemento.

\subsection{Soportes}

No se consideran los soportes mixtos, tal y como se hacía 
TABLA $11(50.2 .2 .1)$

Relaciones $L / d$ en elementos estructurales de hormigón armado sometidos a flexión simple

\begin{tabular}{|c|c|c|}
\hline Sistema estructural & $\begin{array}{c}\text { Elementos } \\
\text { fuertemente armados } \\
\left(\rho=A_{s} b_{0} d=0,012\right)\end{array}$ & $\begin{array}{c}\text { Elementos } \\
\text { débilmente armados } \\
\left(\rho=A_{s} / b_{0} d=0,004\right)\end{array}$ \\
\hline $\begin{array}{l}\text { Viga simplemente apoyada. Losa uni o } \\
\text { bidireccional simplemente apoyada }\end{array}$ & 14 & 20 \\
\hline $\begin{array}{l}\text { Viga continua }{ }^{1} \text { en un extremo. Losa uni- } \\
\text { direccional continua }{ }^{1,2} \text { en un solo lado }\end{array}$ & 18 & 24 \\
\hline $\begin{array}{l}\text { Viga continua }{ }^{1} \text { en ambos extremos. Losa } \\
\text { unidireccional continua }{ }^{1,2}\end{array}$ & 20 & 30 \\
\hline $\begin{array}{l}\text { Recuadros exteriores y de esquina en losa } \\
\text { sobre apoyos aislados }{ }^{3}\end{array}$ & 16 & 22 \\
\hline $\begin{array}{l}\text { Recuadros interiores en losa sobre apoyos } \\
\text { aislados }\end{array}$ & 17 & 25 \\
\hline Voladizo & 6 & 9 \\
\hline
\end{tabular}

1 Un extremo se considera continuo si el momento correspondiente es igual o superior al $85 \%$ del momento de empotramiento perfecto.

2 En losas unidireccionales, las esbelteces dadas se refieren a la luz menor.

${ }^{3}$ En losas sobre apoyos aislados (pilares), las esbelteces dadas se refieren a la luz mayor.

en la EH. En el artículo $1^{\circ}$ de la EHE se excluyen de la misma las estructuras mixtas de hormigón y otro material, sirviendo como referencia técnica el Eurocódigo 4.

\subsection{Placas o losas}

La primera diferencia, aunque sea de tratamiento o presentación, con la EH es que en la misma se encontraba más concentrada la información de estos elementos estructurales, mientras en la EHE se encuentra repartido en los capítulos y artículos correspondientes a Análisis estructural, E.L.U. de Punzonamiento y Elementos estructurales.

Por lo que respecta a aspectos geométricos no se limita el ancho del nervio de los forjados reticulares, estableciendo un espesor mínimo de la capa de compresión de $5 \mathrm{~cm}$, cualquiera que sea el tipo de aligeramiento.

Se modifica sustancialmente la figura correspondiente a disposiciones constructivas, suprimiéndose el caso de "barras levantadas", acotándose desde cara del soporte (y no desde el eje) y cambiando otra serie de aspectos relativos a solape, anclaje y prolongación de armaduras.

\subsection{Elementos de cimentación}

Incluye el tratamiento de losas de cimentación, vigas centradoras y pilotes además de los tradicionales encepados y zapatas.
La clasificación de elementos estructurales se establece dividiéndolos en rígidos $\left(\mathrm{v}_{\text {max }} \leq 2 \mathrm{~h}\right)$ y flexibles $\left(\mathrm{v}_{\text {max }}>2 \mathrm{~h}\right)$.

Para las cimentaciones rígidas (encepados, zapatas, pozos y elementos masivos) se considera como método general de análisis más adecuado el de bielas y tirantes. Para las flexibles (encepados, zapatas y losas) es de aplicación la teoría general de flexión.

\subsection{Vigas de gran canto}

En su análisis no son aplicables las hipótesis de BernouilliNavier, tratándose como una "región D generalizada" mediante el método de bielas y tirantes.

\subsection{Elementos con empuje al vacío}

Son elementos en los que se produce un cambio en la dirección de las fuerzas debido a la geometría del mismo, pudiendo aparecer tracciones transversales que pueden provocar la rotura del recubrimiento. El diseño de la armadura de atado se realizará, tratándose de una región $\mathrm{D}$ siguiendo el método de bielas y tirantes.

\section{EJECUCIÓN}

Sobre el Capítulo XIII, en el que se recogen 15 artículos, subrayaremos sólo aquellos cambios que pueden tener un: mayor incidencia en la práctica habitual. 
15.1. Elaboración de ferralla y colocación de las armaduras pasivas

Se especifica la disposición de separadores para cada tipo de elemento estructural, mediante una distancia máxima entre los mismos.

Para el doblado de armaduras se establecen unos diámetros mínimos de mandriles en función del diámetro de la barra y del tipo de forma generado mediante el citado doblado.

Además de los anclajes mediante prolongación recta y en patilla $\left(90^{\circ}\right)$ de la EH, se contemplan en gancho $\left(\alpha \geq 150^{\circ}\right)$, patilla $\left(90^{\circ} \leq \alpha \leq 150^{\circ}\right)$, gancho en " $U$ " y barra transversal soldada, como se aprecia en la figura 3 (66.5.1), siendo este último la mayor novedad (permite la reducción de la longitud neta de anclaje para barras que trabajan a tracción y a compresión).

Se prohíbe la soldadura de armaduras galvanizadas o con recubrimiento epoxídico, así como hacerlo sobre una superficie que se encuentre a una temperatura inferior a $\operatorname{los} 0^{\circ} \mathrm{C}$.
Se contempla la tipología de empalme mecánico, estableciendo un límite de $0,1 \mathrm{~mm}$ al desplazamiento relativo bajo tensión de servicio y admitiendo la concentración de todos los empalmes en la misma sección.

\subsection{Colocación y tesado de las armaduras activas}

En las armaduras postesas se realiza una pequeña modificación en la separación libre mínima de vainas.

Para la adherencia de las armaduras activas al hormigón, la longitud de transmisión y longitud de anclaje se determinarán, en general, experimentalmente. Sin embargo, en comentarios se presenta una fórmula de estimación de las mismas.

\subsection{Fabricación y transporte a obra del hormigón}

En el artículo $69^{\circ}$ de la EHE se indica que una central de hormigonado (calificación a efectos, entre otros aspectos, de la aplicación del coeficiente $\mathrm{K}_{\mathrm{N}}$ en el control de materiales), puede pertenecer o no a las instalaciones propias de la obra. En cualquier caso, para recibir dicho calificativo ha de cumplir los requisitos prescritos. Sin

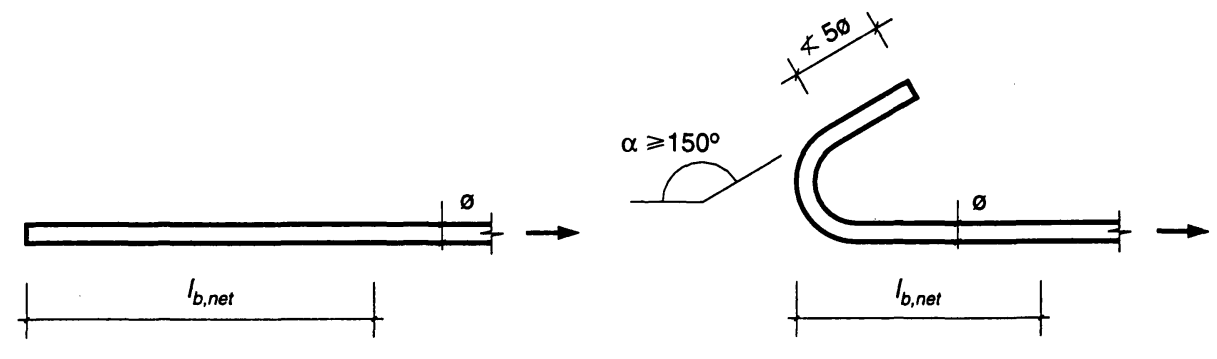

a) PROLONGACIÓN RECTA

b) GANCHO

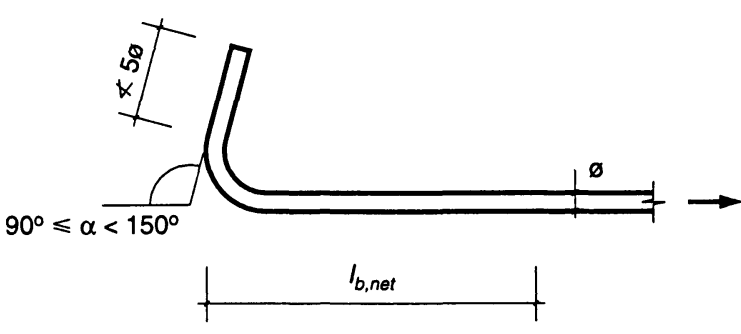

c) PATILLA
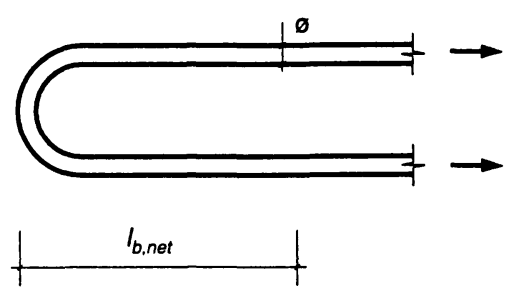

d) GANCHO EN U

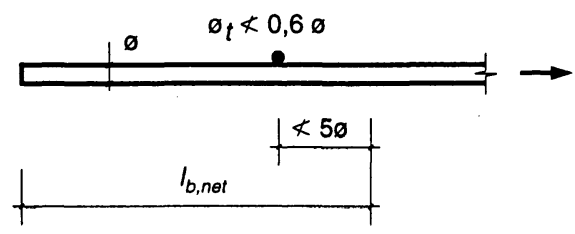

e) BARRA TRANSVERSAL SOLDADA

Figura 3 
embargo, la denominación de "hormigón preparado" sc reserva para aquél que se fabrica en una central que no pertenece a las instalaciones propias de la obra.

En la dosificación del hormigón, la de los áridos se realizará en peso (en EH y EP se admite en peso o en volumen), mientras que la tolerancia de éstos y del cemento pasa a ser de un $\pm 3 \%$.

La designación del hormigón será por propiedades o por dosificación (en EH, por resistencia o por dosificación). En ambos casos se especificará, entre otros puntos, el tipo de ambiente de exposición y el uso (M, A, P).

En la documentación de entrega y recepción se indicará, entre otros y como novedad, el nombre del peticionario y del responsable de la recepción; en la designación por propiedades la tipificación (T-R/C/TM/A), el contenido de cemento $( \pm 15 \mathrm{~kg})$ y la relación agua/cemento $( \pm 0,02)$; en la designación por dosificación el contenido de cemento, la relación agua/cemento $( \pm 0,02)$ y el tipo de ambiente; se deberá mencionar de forma expresa si no contiene ningún aditivo $o$ adición, así como la identificación de la persona que realiza la descarga del camión-hormigonera.

\subsection{Curado del hormigón}

En comentarios se presenta un método de estimación de la duración mínima de curado $\left(D=K . L . D_{0}+D_{1}\right)$ en función de las condiciones ambientales durante el mismo, la velocidad de desarrollo de la resistencia del hormigón (dependiente de la clase de cemento empleado y la relación agua/ cemento), la clase de exposición ambiental, la temperatura media durante el curado y el tipo de cemento empleado.

\subsection{Descimbrado, desencofrado y desmoldeo}

En comentarios, además de la formulación conocida de anteriores instrucciones, se presenta una tabla de períodos mínimos de desencofrado y descimbrado de elementos de hormigón armado para los casos en que no se disponga de datos suficientes para un cálculo más preciso. Los resultados que arroja la mencionada tabla se encuentran en función del tipo de elemento y de la temperatura superficial del hormigón.

\subsection{Inyección}

Antes de su ejecución debe prepararse un programa de inyección en el que se contemplen las características de la lechada y del equipo a emplear, las fases de ejecución y control, así como instrucciones sobre las actuaciones pertinentes si se producen incidentes o condiciones climáticas perjudiciales.

Se establece una velocidad de avance de 5 a $15 \mathrm{~m} / \mathrm{min}$ y una longitud máxima de inyección de $120 \mathrm{~m}$.

\section{BASES GENERALES DEL CONTROLDE CALI- DAD}

Se establece con carácter preceptivo el control de recepción de la calidad del hormigón y de sus materiales componentes.

Se realiza la distinción entre control interno (ejercido por el productor) y control externo (ejercido por el receptor), haciendo referencia el articulado, fundamentalmente, a este último.

En comentarios, se establece una clasificación del control, en función de la tarea controlada, en proyecto, materiales y ejecución.

\section{CONTROLDE MATERIALES}

\subsection{Control de los componentes del hormigón}

Para el hormigón fabricado en central (de hormigón preparado o de obra) no es necesario el control de recepción en obra de materiales componentes si la misma tiene control de producción y Sello o Marca de Calidad, o si el hormigón posee un distintivo reconocido o CC-EHE.

Si no es así, es preciso realizar el mencionado control de materiales, debiendo conservar el responsable de recepción del cemento en la central una muestra por lote durante al menos 100 días y estando firmado el certificado de garantía por persona física.

El certificado de idoneidad de los áridos ha de contar con una antigüedad inferior a 1 año, prestando gran atención durante la obra a la constancia del módulo de finura de la arena.

El certificado de los aditivos ha de encontrarse firmado por persona física, debiendo ensayarse las adiciones antes del comienzo de obra y durante la misma cada tres meses.

\subsection{Control de calidad del hormigón}

Comprenderá, además de la resistencia y consistencia (EP y EH), la durabilidad.

Cada amasada de hormigón fabricado en central estará acompañada por una hoja de suministro cumplimentada y firmada por persona física, que deberá ser archivada por el Constructor.

\subsection{Control de la consistencia y de resistencia del hormigón}

Para el control de la consistencia del hormigón se tomará una muestra de la mitad central (tercio central en EH). 
El control de la resistencia de hormigón se llevará a cabo mediante la rotura de 2 probetas por amasada ( $3 \mathrm{en} \mathrm{EH}$ ).

\subsection{Control de las especificaciones relativas a la durabilidad del hormigón}

Además del control documental a través de las hojas de suministro, que se realizará para todas las amasadas, se llevará a cabo el ensayo de "profundidad de penetración de agua". Este ensayo se llevará a cabo sobre 3 probetas para cada tipo de hormigón, con independencia de la resistencia o consistencia, previo al inicio de la obra.

Para hormigones fabricados en central la Dirección de obra puede eximir del mismo, si el suministrador presenta antes del inicio de la obra la correspondiente documentación que posea una antigüedad inferior a 6 meses. A su vez, si la central es de hormigón preparado (exterior a la obra) con Sello o Marca de Calidad, está eximido de realizar los ensayos, si éstos se encuentran incluidos en su Sistema de Calidad.

\subsection{Ensayos previos y característicos del hormigón}

La novedad fundamental de ambos tipos de ensayo es su realización sobre 2 probetas por serie ( 3 en $\mathrm{EH}$ ).

La relación entre la resistencia media y la característica se establece mediante una nueva expresión $\left(\mathrm{f}_{\mathrm{cm}}=\mathrm{f}_{\mathrm{ck}}+\right.$ $8 \mathrm{~N} / \mathrm{mm}^{2}$ ), independiente de las condiciones previstas para la ejecución de la obra.

\subsection{Ensayos de control del hormigón}

Este es uno de los puntos que más modificaciones, y con más incidencia en la práctica diaria, ha sufrido en la EHE. Frente a la clasificación de la anterior Instrucción en Control total (100\%) y Control estadístico (niveles reducido, normal e intenso), en la presente se realiza directamente en Control a nivel reducido, al $100 \%$ y estadístico, subrayando la importancia de este último.

En la tabla 12 (88.4.a) se muestran los límites máximos establecidos para los lotes. En el "tipo de elementos estructurales" se modifica la definición de los mismos, clasificándose no en elementos comprimidos y elementos en flexión simple (EH), sino en "estructuras que tienen elementos comprimidos" y "estructuras que tienen únicamente elementos sometidos a flexión". En el primer caso se incluyen, por tanto, en el mismo lote los pilares y vigas y forjados de una estructura dada, siempre que aquéllos sean de hormigón.

Todas las unidades de producto (amasadas) de un mismo lote, han de proceder de un mismo suministrador, tener las mismas materias primas y la misma dosificación nominal. A su vez, el número de amasadas por lote se determinará en función de la resistencia característica del hormigón $(\mathrm{N} \geq$ 2 , para $f_{c k} \leq 25 \mathrm{~N} / \mathrm{mm}^{2} ; \mathrm{N} \geq 4$, para $25<\mathrm{f}_{\mathrm{ck}} \leq 35 \mathrm{~N} / \mathrm{mm}^{2}$; $\mathrm{N} \geq 6$, para $\mathrm{f}_{\mathrm{ck}}>35 \mathrm{~N} / \mathrm{mm}^{2}$ ).

TABLA $12(88.4 . a)$

Límites máximos para el establecimiento de los lotes de control

\begin{tabular}{|l|c|c|c|}
\hline \multirow{2}{*}{ Límite superior } & \multicolumn{3}{|c|}{ Tipo de elementos estructurales } \\
\cline { 2 - 4 } & $\begin{array}{c}\text { Estructuras que } \\
\text { tienen elementos } \\
\text { comprimidos } \\
\text { (pilares, pilas, } \\
\text { muros portantes, } \\
\text { pilotes, etc.) }\end{array}$ & $\begin{array}{c}\text { Estructuras que } \\
\text { tienen únicamente } \\
\text { elementos sometidos } \\
\text { a flexión (forjados } \\
\text { de hormigón con } \\
\text { pilares metálicos, } \\
\text { tableros, muros de } \\
\text { contención, etc.) }\end{array}$ & $\begin{array}{c}\text { Macizos (zapatas, } \\
\text { estribos de puente, } \\
\text { bloques, etc.) }\end{array}$ \\
\hline Volumen de hormigón & $100 \mathrm{~m}^{3}$ & $100 \mathrm{~m}^{3}$ & $100 \mathrm{~m}^{3}$ \\
\hline Número de amasadas (1) & 50 & 50 & 100 \\
\hline Tiempo de hormigonado & 2 semanas & 2 semanas & 1 semana \\
\hline Superficie construida & $500 \mathrm{~m}^{2}$ & $1.000 \mathrm{~m}^{2}$ & - \\
\hline Número de plantas & 2 & 2 & - \\
\hline
\end{tabular}

(1) Este límite no es obligatorio en obras de edificación. 
TABLA 13 (88.4.b)

Valores de $K_{N}$

\begin{tabular}{|c|c|c|c|c|c|c|c|c|}
\hline \multirow{4}{*}{$N$} & \multicolumn{7}{|c|}{ Hormigones fabricados en central } & \multirow{4}{*}{$\begin{array}{l}\text { Otros } \\
\text { casos }\end{array}$} \\
\hline & \multicolumn{3}{|c|}{ CLASE A } & \multicolumn{2}{|c|}{ CLASE B } & \multicolumn{2}{|c|}{ CLASE C } & \\
\hline & \multirow{2}{*}{$\begin{array}{l}\text { Recorrido } \\
\text { relativo } \\
\text { máximo, } r\end{array}$} & \multicolumn{2}{|c|}{$K_{N}$} & \multirow{2}{*}{$\begin{array}{l}\text { Recorrido } \\
\text { relativo } \\
\text { máximo, } r\end{array}$} & \multirow[b]{2}{*}{$K_{N}$} & \multirow{2}{*}{$\begin{array}{l}\text { Recorrido } \\
\text { relativo } \\
\text { máximo, } r\end{array}$} & \multirow[b]{2}{*}{$K_{N}$} & \\
\hline & & $\begin{array}{c}\text { Con } \\
\text { Sello de } \\
\text { Calidad }\end{array}$ & $\begin{array}{l}\text { Sin } \\
\text { Sello de } \\
\text { Calidad }\end{array}$ & & & & & \\
\hline $\begin{array}{l}2 \\
3 \\
4 \\
5 \\
6 \\
7 \\
8\end{array}$ & $\begin{array}{l}0,29 \\
0,31 \\
0,34 \\
0,36 \\
0,38 \\
0,39 \\
0,40\end{array}$ & $\begin{array}{l}0,93 \\
0,95 \\
0,97 \\
0,98 \\
0,99 \\
1,00 \\
1,00\end{array}$ & $\begin{array}{l}0,90 \\
0,92 \\
0,94 \\
0,95 \\
0,96 \\
0,97 \\
0,97\end{array}$ & $\begin{array}{l}0,40 \\
0,46 \\
0,49 \\
0,53 \\
0,55 \\
0,57 \\
0,59\end{array}$ & $\begin{array}{l}0,85 \\
0,88 \\
0,90 \\
0,92 \\
0,94 \\
0,95 \\
0,96\end{array}$ & $\begin{array}{l}0,50 \\
0,57 \\
0,61 \\
0,66 \\
0,68 \\
0,71 \\
0,73\end{array}$ & $\begin{array}{l}0,81 \\
0,85 \\
0,88 \\
0,90 \\
0,92 \\
0,93 \\
0,95\end{array}$ & $\begin{array}{l}0,75 \\
0,80 \\
0,84 \\
0,87 \\
0,89 \\
0,91 \\
0,93\end{array}$ \\
\hline
\end{tabular}

Para la determinación del coeficiente " $\mathrm{K}_{\mathrm{N}}$ ", que también se ha modificado, se utilizará la tabla 13 (88.4.b). Dicho valor es función del número de amasadas por lote y del tipo de planta de fabricación de hormigón (o del recorrido relativo máximo " $r$ " de los resultados de los ensayos en cada lote).

Respecto a las decisiones derivadas del control de la resistencia a compresión y, en el caso de obtener una $f_{c, e s t}<0,9 f_{c k}$, se procederá a realizar una serie de estudios complementarios. Entre ellos se encuentra la prueba de carga en la que, frente a la indicación de la EH de que la carga de ensayo no excederá del valor característico de la carga tenida en cuenta en el cálculo, en la EHE se indica que podrá exceder dicho valor, recomendando una carga total materializada de un $85 \%$ de la carga total de cálculo.

\subsection{Control de la calidad del acero}

Los niveles de control, que eran tres (reducido, normal e intenso) en la EH y los dos últimos en la EP, quedan en dos (reducido y normal) en la EHE, no admitiéndose (como en la EP) el control reducido para acero de armadura activa.

Todas las partidas de acero han de estar acompañadas del certificado de garantía del fabricante, firmado por persona física.

Respecto al certificado de calidad del acero, su influencia se manifiesta, fundamentalmente, en dos aspectos: fase de realización de ensayos y número de éstos. Los ensayos del acero (con resultado favorable) han de encontrarse realizados antes del hormigonado del elemento estructural cuando se trate de un acero sin certificación, y antes de la puesta en servicio de la estructura cuando sea certificado.
El control a nivel reducido sólo se admite para armaduras pasivas, con acero certificado y reduciendo en un $25 \%$ el límite elástico de cálculo.

El control normal será aplicable a armaduras activas y pasivas, clasificándose esta última en tres series (fina, media y gruesa) en función del diámetro de las barras corrugadas. Los lotes, para el mismo suministrador, designación y serie, serán de 40 y 20 t, respectivamente, para acero certificado y no certificado de armadura pasiva y de 20 y $10 \mathrm{t}$, para la armadura activa. Se tomarán dos probetas por serie.

Se contempla la comprobación de la soldabilidad, además de sobre la soldadura a tope (como en EH), sobre las soldaduras por solapo y en cruz.

Deberá tenerse en cuenta que para obras de edificación desaparecen las consideraciones especiales del sellc CIETSID.

\section{CONTROLDE LA EJECUCIÓN}

\subsection{Control de la ejecución}

Para desarrollar este control se divide la obra en los lotes indicados en la tabla 14 (95.1.a), revisando en cada uno de ellos los aspectos recogidos en las relaciones que figuran en el articulado de la EHE, clasificando los mismos, básicamente, en generales para todo tipo de obra, específicos para forjados de edificación y propios de prefabricación.

Los tres niveles de control de ejecución, intenso, normal y reducido, se encuentran íntimamente ligados a los coefi- 
TABLA $14(95.1 . a)$

\begin{tabular}{|l|l|}
\hline \multicolumn{1}{|c|}{ Tipo de obra } & \multicolumn{1}{c|}{ Tamaño del lote } \\
\hline Edificios & $500 \mathrm{~m}^{2}$, sin rebasar las dos plantas \\
\hline Puentes, Acueductos, Túneles, etc. & $500 \mathrm{~m}^{2}$ de planta, sin rebasar los $50 \mathrm{~m}$ \\
\hline Obras de Grandes Macizos & $250 \mathrm{~m}^{3}$ \\
\hline Chimeneas, Torres, Pilas, etc. & $250 \mathrm{~m}^{3}$, sin rebasar los $50 \mathrm{~m}$ \\
\hline $\begin{array}{l}\text { Piezas prefabricadas: } \\
\text { - De tipo lineal }\end{array}$ & $\begin{array}{l}500 \mathrm{~m} \text { de bancada } \\
\text { - De tipo superficial }\end{array}$ \\
\hline
\end{tabular}

cientes de mayoración de las acciones, tal y como se puede apreciar en la tabla 5 (12.1.b).

El control a nivel intenso establece los siguientes requisitos:

-Control externo con tres inspecciones por lote.

-El Constructor ha de poseer un Sistema de Calidad propio, auditado de forma externa.

-La elaboración de la ferralla y los elementos prefabricados se ha de realizar en instalaciones industriales fijas y con un sistema de certificación voluntario.

Los niveles de control normal y reducido requieren, respectivamente, dos y una inspección por lote.

\subsection{Tolerancias de ejecución}

En esta Instrucción se introducen, por vez primera, las tolerancias en la ejecución, que se plantea como un sistema de complemento de lo indicado en el Pliego de Condiciones del Proyecto.

En el Anejo $n^{\circ} 10$ se desarrollan, de una forma concreta, las tolerancias para cada tipo de elemento estructural o unidad constructiva.

\subsection{Pruebas de carga}

Las pruebas de carga se clasifican, según su finalidad, en reglamentarias, de información complementaria y de evaluación de la capacidad portante.

Las pruebas de carga reglamentarias son las fijadas por los Pliegos, Instrucciones o Reglamentos para puentes de carretera o de ferrocarril, así como las dinámicas preceptivas en casos específicos.
Las pruebas de carga como información complementaria, motivada por cambios o problemas durante la ejecución, no deben sobrepasar las acciones de servicio con carácter general y los criterios de aceptación o rechazo han de ser semejantes a las anteriores.

Las pruebas de carga para evaluar la capacidad resistente de una estructura o elemento estructural han de contar con un plan de prueba, encontrando su aplicación fundamental en elementos sometidos a flexión.

\section{ANEJOS}

Los anejos de la presente Instrucción se pueden clasificar, en función de las modificaciones producidas, en tres tipos: los que mantienen el mismo esquema y filosofia, aun con algún cambio, los que han tenido alteraciones sustanciales y los que se presentan como nuevos, sin que figuraran en las anteriores Instrucciones. En este apartado se realiza un resumen de los aspectos más significativos de estos dos últimos.

\subsection{Utilización de los cementos}

El Anejo $n^{0} 3$, "Recomendaciones generales para la utilización de los cementos especificados en la Instrucción para la Recepción de Cementos RC-97", incorpora los cambios producidos en la citada Instrucción, de publicación posterior a la EH y la EP.

En la EHE se presentan tres cuadros cuya intersección arroja el tipo de cemento recomendable en función de las aplicaciones del hormigón, las circunstancias de hormigonado y las clases de exposición en servicio. Se incluyen unos ejemplos de aplicación.

\subsection{Cemento de aluminato de calcio}

En el Anejo n ${ }^{\circ} 4$, "Prescripciones para la utilización del cemento de aluminato de calcio", se aportan una serie de 
modificaciones respecto al correspondiente anejo precedente, ampliándose la información sobre las características de este material.

Se limita la resistencia característica del hormigón fabricado con él a $40 \mathrm{~N} / \mathrm{mm}^{2}$, estableciéndose unos recubrimientos mínimos en función de la clase de exposición del elemento estructural y aportando una relación de aplicaciones concretas del mismo.

\subsection{Agresividad de agua y suelo}

En el Anejo no 5, "Métodos de ensayo para determinar la agresividad de aguas y suelos al hormigón", que se presenta como novedad de esta Instrucción, se describen los procedimientos para la toma de muestras, la realización de los ensayos químicos (análisis de aguas y de suelos) y la presentación de resultados.

\subsection{Protección contra el fuego}

El Anejo $n^{\circ}$ 6, "Recomendaciones para la protección adicional contra el fuego de elementos estructurales", es una ampliación del correspondiente anejo de la anterior $\mathrm{EH}$.

Se presentan cuatro métodos de comprobación: general, simplificado, mediante tablas y mediante ensayos, indicando, específicamente, que no son de aplicación el segundo y el tercero para estructuras laminares ni para pretensado exterior. Para los hormigones de alta resistencia se desarrollan unas disposiciones adicionales.

En el anejo se presenta con más detalle la comprobación mediante tablas para hormigones con densidad normal y con áridos silíceos. Para cada elemento estructural analizado (pilares, muros, tirantes, vigas, losas macizas, losas nervadas y forjados reticulares), se establecen unas dimensiones y unos recubrimientos mecánicos equivalentes mínimos. Se considera, de forma adicional e independiente, el efecto de las capas protectoras.

\subsection{Estado Límite de Agotamiento frente a solicita- ciones normales}

El Anejo $n^{\circ} 8$, "Cálculo simplificado de secciones en Estado Límite de Agotamiento frente a solicitaciones normales", presenta las fórmulas simplificadas para el cálculo (dimensionamiento y comprobación) de secciones rectangulares o en "T" sometidas a flexión simple o compuesta recta, así como el método simplificado de reducción a flexión compuesta recta de secciones sometidas a flexión esviada simple o compuesta.

La principal aportación es la modificación del método de cálculo simplificado del momento tope que figuraba en la EH.

\subsection{Fisuración}

El Anejo n ${ }^{\circ}$, "Análisis de secciones fisuradas en servicio sometidas a flexión simple", es nuevo en la presente Instrucción.

Las expresiones presentadas en este anejo permiten determinar las tensiones en la armadura traccionada para la comprobación del Estado Límite de Fisuración, así como evaluar la inercia fisurada para la comprobación del Estado Límite de Deformaciones:

\subsection{Tolerancias}

Este Anejo $n^{\circ} 10$ de la EHE, que se incorpora como novedad en la misma, indica que el sistema de tolerancias debe quedar establecido en el proyecto en el Pliego de Prescripciones Técnicas Particulares, ya sea por referencia al mencionado anejo, ya sea completado o modificado. Se indica expresamente que las tolerancias adoptadas deben ser las más amplias, compatibles con el funcionamiento adecuado de la construcción.

El sistema de este anejo está previsto para obras de hormigón de tipo usual, debiendo adaptarse a cada proyecto concreto.

Se indican los principios generales seguidos y las desviaciones admisibles en armaduras, cimentaciones, elementos de estructuras de edificios construidos in situ, piezas prefabricadas (excluidos pilotes), pantallas, núcleos, torres, chimeneas, pilas y otros elementos hormigonados con encofrado deslizante, muros de contención y muros de sótano, obras hidráulicas y sanitarias (canales, alcantarillas, sifones...), puentes y estructuras análogas hormigonadas in situ, pavimentos y aceras (excluidas las carreteras, que disponen de especificaciones propias) y obras civiles de elementos de gran espesor, no incluidas en otros apartados.

\subsection{Hormigón de alta resistencia}

En el Anejo n ${ }^{\circ} 11$, "Recomendaciones para hormigones de alta resistencia", se definen como tales aquellos que tienen una resistencia característica de proyecto superior a los $50 \mathrm{~N} / \mathrm{mm}^{2}$ e igual o inferior a los $100 \mathrm{~N} / \mathrm{mm}^{2}$.

Por ser nuevo en la presente Instrucción, su conocimiento requiere una lectura detenida. Sin embargo, se pueden señalar los siguientes como algunos de sus aspectos más significativos:

-Su dosificación requiere otros métodos distintos a los contemplados en hormigones nørmales.

-El contenido de cemento se establece entre 400 y 500 $\mathrm{kg} / \mathrm{m}^{3}$, la relación agua/cemento ha de ser inferior a 0,40 
y el porcentaje de humo de sílice (adición especialmente indicada para este hormigón) se sitúa entre el 8 y el $12 \%$ del contenido de cemento.

-El control de la resistencia a compresión se realizará mediante probetas cilíndricas de $\phi 100 \mathrm{~mm}$ o cúbica, de $100 \mathrm{~mm}$ de arista, con un coeficiente de conversión entre ambas (para $70<\mathrm{f}_{\mathrm{c}}<90 \mathrm{~N} / \mathrm{mm}^{2}$ ) de 0,85 a 0,90.

-Es un hormigón menos permeable, con mejor resistencia a los ciclos de hielo-deshielo, con mayor riesgo de reacción árido-álcali, con mejor protección frente a los fenómenos de corrosión de armaduras y más susceptible a la acción del fuego.

-Para su tipificación se recomienda la serie: H-55, H-60, $\mathrm{H}-70, \mathrm{H}-80, \mathrm{H}-90$ y $\mathrm{H}-100$.

-La puesta en obra se lleva a cabo, habitualmente, mediante bombeo, con tongadas que pueden ser de mayor espesor, necesitando mayor energía de compactación, para una misma consistencia, que un hormigón normal y presenta menor segregación durante el vertido.

-El curado debe cuidarse especialmente, sobre todo, los tres primeros días tras el hormigonado.

-Respecto al cálculo estructural, presenta ciertas particularidades, tanto en lo relativo a la comprobación de los Estados Límite Últimos como a los Estados Límite de Servicio.

-El control de materiales presenta una serie de ensayos adicionales sobre el cemento y los áridos, estableciéndose el del hormigón según unos lotes, en función del tipo de elementos estructurales.

\subsection{Acciones sísmicas}

El Anejo no 12, "Requisitos especiales recomendados para estructuras sometidas a acciones sísmicas", describe los que se recomiendan para estructuras sometidas a dichas acciones, complementarios a las disposiciones establecidas en la Norma de construcción sismorresistente española NCSE-94.

Los métodos de análisis estructural que se establecen son el estudio dinámico directo, el análisis modal y el método simplificado de cargas estáticas equivalentes.

Los aceros de armaduras pasivas recomendados para garantizar un comportamiento de ductilidad elevada son los que cumplen las condiciones de $1,20 \leq \mathrm{f}_{\mathrm{s}} / \mathrm{f}_{\mathrm{y}} \leq 1,35$ y $\varepsilon_{\text {máx }} \geq 9 \%$. Un tipo de acero que cumple estas condiciones es el B $400 \mathrm{SD}$, constituido por barras corrugadas cuyos diámetros son $6,8,10,12,14,16,20,25,32$ y 40 . Sus requisitos técnicos son los de soldabilidad, adherencia, características mecánicas recogidas en la tabla 15(A.12.1), resistencia a la fatiga y resistencia a carga cíclica de gran amplitud.

Se establecen unos criterios generales para el dimensionamiento y disposiciones constructivas de los distintos elementos estructurales, así como unos criterios específicos para vigas y soportes de muy alta y de alta ductilidad y para nudos, pantallas y diafragmas horizontales.

\subsection{Eurocódigo 2}

El Anejo n ${ }^{\circ} 13$, "Documento nacional de aplicación de la norma UNE ENV 1992-1-1 experimental”, que es nuevo en la presente Instrucción, tiene por objetivo el estableci-

TABLA 15(A.12.1)

Características mecánicas garantizadas de las barras corrugadas de acero B 400 SD

\begin{tabular}{|c|c|c|c|c|c|c|c|}
\hline Designación & $\begin{array}{c}\text { Clase } \\
\text { de } \\
\text { acero }\end{array}$ & $\begin{array}{c}f_{y}\left[\mathrm{~N} / \mathrm{mm}^{2}\right] \\
\text { no menor } \\
\text { que (1) }\end{array}$ & $\begin{array}{c}f_{s}\left[\mathrm{~N} / \mathrm{mm}^{2}\right] \\
\text { no menor } \\
\text { que (1) }\end{array}$ & $\begin{array}{c}f_{y} \text { real / } \\
f_{y} \text { nominal }\end{array}$ & $\begin{array}{c}\text { Alargamiento } \\
\text { de rotura } \\
\text { sobre } \\
\text { base de } \\
\text { diámetros }\end{array}$ & $\begin{array}{c}f_{s} / f_{y} \\
\text { en } \\
\text { ensayo } \\
(2)\end{array}$ \\
\hline B 400 SD & $\begin{array}{c}\text { Soldable } \\
\text { con } \\
\text { características } \\
\text { especiales } \\
\text { de ductilidad }\end{array}$ & 400 & 480 & $\leqslant 1,20$ & $\geqslant 20 \%$ & $\geqslant 9 \%$ & $\leqslant 1,35$ \\
\hline
\end{tabular}

(1) Para el cálculo de los valores unitarios se utilizará la sección nominal.

(2) Relación mínima y máxima admisible entre la carga unitaria de rotura y el límite elástico obtenido en cada ensayo. 
miento de unas condiciones que hagan viable la aplicación de la citada norma al proyecto y ejecución de estructuras de hormigón, a pesar de la carencia de ciertas normas europeas sobre materiales y otros aspectos relacionados con aquélla.

Recoge una serie de valores en recuadro que pueden ser modificados y sustituidos por los de la normativa propia, figurando de este modo los recomendados.

\section{BIBLIOGRAFÍA}

(1) E.T.S. de Ingenieros de Caminos, Canales y Puertos: Universidad Politécnica de Madrid, "Curso sobre la nueva Instrucción de Hormigón Estructural EHE”, Febrero de 1999.
(2) Ministerio de Fomento: "Instrucción para la recepción de cementos, RC-97”, 1997.

(3) Ministerio de Fomento: "Instrucción de Hormigón Estructural EHE”, 1998.

(4) Ministerio de Obras Públicas, Transporte y Medio Ambiente: "Instrucción para el proyecto y la ejecución de obras de hormigón en masa o armado, EH-91”, 1991

(5) Ministerio de Obras Públicas, Transporte y Medio Ambiente: "Instrucción para el proyecto y ejecución de obras de hormigón pretensado, EP-93", 1993.

(6) Ministerio de Obras Públicas, Transporte y Medio Ambiente: "Instrucción para el proyecto y la ejecución de forjados unidireccionales de hormigón armado o pretensado, EF-96", 1996.

\section{Publicaciones del Instituto Eduardo Torroja-CSIC}

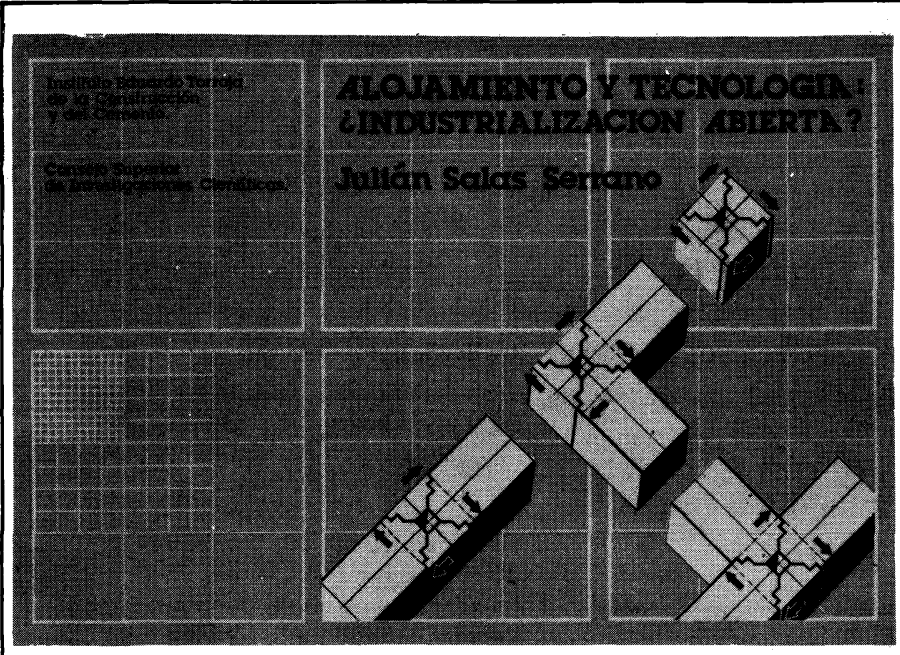

ALOJAMIENTO Y TECNOLOGIA: ¿INDUSTRIALIZACION ABIERTA?

JULIAN SALAS, ING. IND. (I.E.T.c.c.)

Un volumen de 160 páginas, 109 figuras y 16 tablas. Tamaño $240 \times 168 \mathrm{~mm}$. Encuadernado en rústica.

\section{SUMARIO:}

Prólogo Prof. G. Ciribini.

\section{Introducción}

Capítulo 1. - La industrialización en las proclamas y manifiestos de arquitectura.

Capitulo 2. ¿Réquiem por la construcción industrializada?

Capítulo 3.-Algunos conceptos básicos.

Capitulo 4.-¿Proyecto tradicional, construcción industrializada?

Capítulo 5. - Componentes.

Capitulo 6. - La coordinación dimensional hoy.

Capítulo 7.-Flexibilidad, intercambiabilidad y catálogos.

Capítulo 8. - Industrialización, normativa y calidad.

Capítulo 9. - Reflexiones finales.

publicación del

INSTITUTO EDUARDO TORROJA 